\title{
Androgen receptor and prostate apoptosis response factor-4 target the c-FLIP gene to determine survival and apoptosis in the prostate gland
}

\author{
Shen Gao, Hua Wang, Peng Lee ${ }^{1}$, Jonathan Melamed ${ }^{1}$, Caihong X Li ${ }^{1}$, Fahao Zhang, \\ Hong Wu, Liran Zhou and Zhengxin Wang
}

Department of Cancer Biology, The University of Texas M D Anderson Cancer Center, 1515 Holcombe Boulevard, Houston, Texas 77030, USA

${ }^{1}$ Department of Pathology, New York University School of Medicine, New York, New York 10010, USA

(Requests for offprints should be addressed to Z Wang; Email: zhenwang@mdanderson.org)

\begin{abstract}
Androgen receptor (AR) is a ligand-activated transcription factor that mediates the action of androgens and is essential for the growth, function, and cell differentiation of the prostate gland. Here, we demonstrated that the prostate apoptosis response factor-4 (par-4) functions as a novel AR coactivator. Par-4 physically interacted with the DNA-binding domain of $A R$, enhanced $A R$ interaction with DNA, and increased AR-dependent transcription. Par-4 enhanced the c-FLIP promoter activity and was recruited on to the c-FLIP gene in the presence of androgens, and the dominant-negative par-4 decreased c-FLIP expression. These results suggest that, in addition to its proapoptotic function, par-4 acts as a novel transcription cofactor for AR to target c-FLIP gene expression. In addition, we demonstrated that loss of c-FLIP expression was essential for castration-induced apoptosis in the prostate gland and that enhanced c-FLIP expression was associated with prostate cancer progression to the androgen-resistant stage. Our data shed light on a transcription-mediated mechanism for the effects of the AR pathway on cell survival and apoptosis.
\end{abstract}

Journal of Molecular Endocrinology (2006) 36, 463-483

\section{Introduction}

Androgens play a pivotal role in maintaining prostate homeostasis (Denmeade et al. 1996) and under abnormal conditions they contribute to the development of prostate cancer (Bentel \& Tilley 1996, Craft et al. 1999). The androgen receptor (AR) mediates the action of androgens and is a ligand-inducible transcription factor containing a central DNA-binding domain (DBD), a C-terminal ligand-binding domain (LBD), and an N-terminal domain (NTD) (Hakimi et al. 1996, Brinkmann et al. 1999, Gelmann 2002). Upon androgen binding, AR regulates the transcription of specific target genes by binding to specific DNA response elements, androgen response elements (AREs), in their promoters. After binding DNA, AR interacts with components of the basal transcription machinery and a variety of general and gene-specific cofactors, resulting in positive or negative effects on gene transcription (Janne et al. 2000, Gelmann 2002, Heinlein \& Chang 2002). A panel of proteins that can interact with the $\mathrm{AR}$ have been identified (ww2.mcgill.ca/androgendb/ARinteract.pdf). These include $\mathrm{p} 300 / \mathrm{CREB}$ binding protein (CBP), the p160 family, p300/CREB binding protein associated factor/General control of amino acid synthesis protein
(PCAF/GCN5) complexes, the mediator complex, the androgen receptor associated protein (ARA) group, and various other proteins. However, the details of the molecular mechanisms, gene specificity, and biological relevance of these cofactors in AR-driven transcription remain unclear. We and others demonstrated nearly constant expression of $\mathrm{AR}$ and heterogeneous expression of AR cofactors in primary prostate cancer (NesslerMenardi et al. 2000, Li et al. 2002, Miyoshi et al. 2003, Culig et al. 2004), suggesting that dysregulated expression of cofactors might be important for modulating $\mathrm{AR}$ function in prostate tumorigenesis.

The prostate apoptosis response factor- 4 (par-4) gene was originally identified by differential screening for genes that are up-regulated when prostate cells are induced to undergo apoptosis (Sells et al. 1994). Par-4 was subsequently found to possess potent apoptotic activity in various cellular systems in response to numerous stimuli (Mattson et al. 1999, Moscat \& Diaz-Meco 2003, Gurumurthy \& Rangnekar 2004). Part of this effect has been proposed to result from activation of the Fas/FasL-mediated apoptosis pathway (Chakraborty et al. 2001). More recently, functional and genetic analyses indicated that par-4 functions as a negative regulator of the protein kinase $\mathrm{C}$-nuclear 
factor- $\kappa \mathrm{B} \quad(\mathrm{NF}-\kappa \mathrm{B})-\mathrm{X}$-linked inhibitor of apoptosis protein (XIAP) pathway (Garcia-Cao et al. 2003, Moscat \& Diaz-Meco 2003). In addition, par-4 has also been identified as a transcription cofactor for the Wilms' tumor suppressor gene (WT1) through direct interaction with the zinc-finger DBD of WT1 (Johnstone et al. 1996, Richard et al. 2001). Par-4 transcriptionally regulates bcl-2 gene expression through a WT1-binding site on the bcl-2 promoter (Boehrer et al. 2002, Cheema et al. 2003). The open-reading frame of human par-4 predicts a protein of 342 amino acids containing two putative nuclear localization signals at the $\mathrm{N}$-terminus and a leucine zipper domain in the C-terminus region of the protein (El-Guendy \& Rangnekar 2003). The C-terminal leucine zipper domain is necessary for homodimerization and heterodimerization with WT1 (Johnstone et al. 1996), protein kinase G (Diaz-Meco et al. 1996), Dlk (Kogel et al. 1998), and p62 (Dutta et al. 2001). Deletions of the leucine zipper domain abolishes its apoptotic activity (Sells et al. 1997, Guo et al. 1998), suggesting that the domain may play a significant role in par-4mediated apoptosis. Recently, a unique core domain containing amino acid residues 137-195 of par-4 was found to be essential and sufficient for induction of apoptosis in some cancer cells (El-Guendy et al. 2003).

In the present study, we found that par-4 directly interacted with the $\mathrm{DBD}$ of $\mathrm{AR}$ and increased AR-dependent transcription by enhancing AR-ARE interaction. We identified the c-FLIP gene as a target of par-4 and found that loss of c-FLIP expression was essential for castration-induced apoptosis in mouse prostate epithelial cells. These results suggest that AR and par-4 target the c-FLIP gene to control survival and apoptosis in the prostate gland.

\section{Materials and methods}

\section{Production of recombinant proteins and antibody production}

An expressed sequence tag cDNA clone (IMAGE 664597) encoding the full-length human par-4 gene was obtained from the American Type Culture Collection (Manassas, VA, USA). All par-4 cDNA fragments were amplified by PCR with specific oligonucleotides, cut with NdeI and BamHI, and subsequently cloned in the corresponding restriction sites of the vectors pET15d (Novagene, Madison, WI, USA), pGEX-2TL (Amersham Biosciences, Piscataway, NJ, USA), and pcDNA3.1 (Invitrogen, Carlsbad, CA, USA). The fragments were expressed as 6 His-tagged (via pET15d) or glutathione S transferase (GST)-fusion (via pGEX2TL) proteins in Escherichia coli BL21 and purified through NTA $\mathrm{Ni}^{2+}$ agarose or glutathione Sepharose columns respectively. The cDNA encoding the c-FLIP short form was subcloned into pET15d, expressed, and purified through NTA $\mathrm{Ni}^{2+}$ agarose in a similar manner. Ten milligrams purified recombinant 6 His-par- 4 and 6 His-c-FLIP (short form) were sent to Sigma Genosis (The Woodlands, TX, USA) for polyclonal antibody production. Anti-6 His-par-4 and anti-6 His-c-FLIP (short form) antibodies were purified through 6 His-par-4- and 6 His-c-FLIP (short form)-affinity columns respectively.

\section{Establishment of prostate cell lines that stably expressed a FLAG-tagged par-4 and the $\mathrm{N}$-terminal peptide of par-4}

The mammalian expression vector, pBabe-f:par-4, was created by subcloning the FLAG-tagged human par-4 cDNA into the vector pBabe-Neo. The pBabe-internal ribosome entry site (IRES)-enhanced green fluorescent protein (EGFP) was constructed by subcloning the EcoRI-SalI fragment of the MSCV MIGR1 vector (Pear et al. 1998) into the EcoRI-SalI site of pBbabe-Neo. The cDNA encoding the N-terminal peptide (amino acid residues 1-36) of par-4 was subcloned into pBabe-IRES-EGFP to generate the pBabe-par-4(1-36)-IRES-EGFP construct. The prostate cancer cell line LNCaP was purchased from the American Type Culture Collection and maintained in RPMI 1640 medium plus 10\% fetal bovine serum. Cells were transfected with pBabe-f:par-4, pBabe-IRESEGFP, or pBabe-par-4(1-36)-IRES-EGFP and further incubated at $37^{\circ} \mathrm{C}$ for $1-1.5$ days before being split 1:6 for G418 selection $(0.5 \mathrm{mg} / \mathrm{ml})$. The medium was changed every 3 or 4 days. Individual G418-resistant colonies were expanded into cell lines and then characterized by western blotting using the anti-FLAG M2 monoclonal antibody (Sigma) for the FLAG-tagged par-4 cell lines or by observation of EGFP under a fluorescent microscope for EGFP and par-4(1-36)IRES-EGFP cell lines. The positive cell lines were further expanded and analyzed. Nuclear and cytoplasmic extracts were prepared according to our standard methods (Wang \& Roeder 1997) and used to immunopurify the par-4-containing complexes as previously described (Hosohata et al. 2003). Five microliter aliquots of the immunopurified complexes were mixed with equal volumes of $2 \times$ Laemmli's sample buffer and loaded onto a $10 \%$ sodium dodecyl sulfate (SDS)polyacrylamide gel. Proteins were visualized by silver staining.

\section{Gel filtration chromatography and gel shift assay}

Fifty microliters f:par-4 immunoprecipitate were loaded onto a Superose 6 column $(3 \cdot 2 \times 300 \mathrm{~mm}$; Smart System, Amersham Biosciences), and the column was eluted with BC300-0.01\% NP-40 at a flow rate of $40 \mu \mathrm{l} / \mathrm{min}$. One hundred microliter fractions were 
collected. High-range gel filtration molecular weight markers (Amersham Biosciences) were separated on the same column under the same conditions. The gel shift assay was performed as previously described (Liu et al. 2003). In brief, the wild-type (ttgcAGAACAGGAAGT GCTagct) and mutant (ttgcAGAAtAGCAAaTGCT agct) ARE probes were derived from the prostatespecific antigen gene ( -152 to -174$)$ and labeled with $\left[\alpha-{ }^{32} \mathrm{P}\right] \mathrm{dCTP}$ by a fill-in reaction with the Klenow enzyme. In gel shift assays, $20 \mu \mathrm{l}$ reaction mixture contains $20 \mathrm{mM}$ HEPES, $\mathrm{pH} 7 \cdot 9,70 \mathrm{mM} \mathrm{KCl}, 1 \mu \mathrm{g}$ poly (dI-dC), $1 \mathrm{mM}$ dithiothreitol, $0 \cdot 1 \%$ NP-40, $100 \mu \mathrm{g} / \mathrm{ml}$ bovine serum albumin, and various proteins. The reaction mixture was incubated for $20 \mathrm{~min}$ at room temperature, and the binding reaction was initiated by the addition of the labeled probes (20000 c.p.m.) and then incubated for an additional $30 \mathrm{~min}$ at room temperature. The reaction mixture was loaded directly onto a 4\% (37.5:1, acrylamide:bisacrylamide) nondenaturing polyacrylamide gel with $0.25 \times$ Tris-borateEDTA and run at $150 \mathrm{~V}$ for $2 \mathrm{~h}$ at ambient temperature.

\section{Cell culture and DNA transfection}

PC3 cells were purchased from the American Type Culture Collection and cultured in RPMI 1640 medium containing $10 \%$ fetal bovine serum. Cells $\left(5 \times 10^{4}\right)$ were plated in each well of 24-well plates and transfected with 25 fmoles luciferase reporter plasmids, $0 \cdot 8$ fmoles control plasmid pRL-GMV, and 50 fmoles expression plasmids (balanced with the empty vector pcDNA3 1 ) using $1 \cdot 5 \mu \mathrm{l}$ Lipofectamine reagent (Invitrogen). Cells were grown in the presence or absence of ligands for $48 \mathrm{~h}$ after transfection and harvested for dual-luciferase activity assay (Promega, Madison, WI, USA).

\section{Protein-protein pull-down assay}

GST and GST-par-4 variant proteins were expressed in bacteria and immobilized on glutathione Sepharose beads (Amersham Biosciences). Beads (10 $\mu$ l) containing $1 \mu \mathrm{g}$ GST or GST-par-4 variant proteins were incubated with $5 \mu \mathrm{l}$ coupled transcription and translation (TNT) rabbit reticulocyte lysates containing ${ }^{35} \mathrm{~S}$-labeled proteins in BC150-0.1 NP-40 for $2 \mathrm{~h}$ at $4{ }^{\circ} \mathrm{C}$. After being washed with the incubation buffer, beads were boiled with SDS sample buffer and subjected to SDSpolyacrylamide gel electrophoresis (PAGE), followed by autoradiography.

\section{Northern blot analysis and chromatin immunoprecipitation}

The northern blot and chromatin immunoprecipitation assays were performed as described (Gao et al. 2005). In brief, mRNAs were isolated from LNCaP cells cultured with phenol red-free RPMI 1640 supplemented with $10 \%$ charcoal/dextran-stripped fetal bovine serum for 2 days and then with or without $10 \mathrm{nM} \mathrm{R} 1881$ for $24 \mathrm{~h}$. For northern blot analysis, $3 \mu \mathrm{g}$ mRNA were fractionated with $1 \%$ denatured formaldehyde-agarose gel, transferred to a Hybond $\mathrm{N}^{+}$membrane (Amersham Biosciences), and cross-linked with ultraviolet light. The 771-bp fragment (729-1499) of human c-FLIP cDNA (Gene Bank U97074) and human $\beta$-actin cDNA were labeled with $\left[\alpha_{-}{ }^{32} \mathrm{P}\right] \mathrm{dCTP}$. Membranes were hybridized with probes at $65^{\circ} \mathrm{C}$ overnight. After being washed to a final stringency of $0 \cdot 1 \times$ SSC- $0 \cdot 1 \%$ sodium dodecyl sulfate (SDS) at $65{ }^{\circ} \mathrm{C}$, the membrane was exposed to X-ray film. For chromatin immunoprecipitation, LNCaP cells were grown in phenol red-free RPMI 1640 supplemented with $10 \%$ charcoal/dextran-stripped fetal bovine serum for 3 days and then treated with $10 \mathrm{nM}$ R1881 for $36 \mathrm{~h}$. Cells treated with ethanol were used as the control. Chromatin immunoprecipitation was performed with $2 \mu \mathrm{g}$ antigen-purified anti-par-4 antibody. Immunopurified DNA was used for a PCR reaction (30 cycles, annealing at $50{ }^{\circ} \mathrm{C}$ ), with the following primers: for c-FLIP, the forward primer sequence was GATCACGAGGTCAAGAGTTC and the reverse primer sequence was GACACAAGTTCG ACGCGTTAG, which amplifies a 290-bp product from -321 to -32 upstream of the c-FLIP gene; for $\beta$-actin, the forward primer sequence was TCCTCGTCTTC CTCAATCTCG and the reverse primer sequence was AAGGGAA CTTTCGGAAGGG, which amplifies a 145-bp product from -1118 to -974 of the $\beta$-actin gene (the A of the ATG translation start codon was arbitrarily given the number +1 ).

\section{Castration-induced apoptosis in the prostate gland}

Twenty male G57BL/6J mice (2 months old) were purchased from The Jackson Laboratory (Bar Harbor, ME, USA) and treated in accordance with the approved protocol of the Institutional Animal Care and Use Committee. Mice were castrated or sham-operated under anesthesia. At each of 2, 7, 15, and 30 days after surgery, prostate glands were dissected, fixed in $10 \%$ formalin overnight, and embedded in paraffin. Paraffin sections $(5 \mu \mathrm{m}$ thick) were cut and mounted on glass slides. After being de-waxed, slides were stained with $0 \cdot 1 \%$ Hill's hematoxylin \#3 and eosin (H\&E) or used for immunohistochemical staining or in a terminal deoxynucleotidyl transferase-mediated dUPT nick-end labeling (TUNEL) assay.

\section{Western blot and immunohistochemical staining}

Whole-cell lysates were electrophoresed on SDS-PAGE and then transferred to a nitrocellulose membrane for 
Western blot analysis. Blots were incubated with anti-AR (1:5000), anti-par-4 (1:5000), anti-c-FLIP (1:5000), or anti-FLAG $(1: 1000)$ (Sigma) antibody for $2 \mathrm{~h}$ at room temperature and then incubated for $1.5 \mathrm{~h}$ with the peroxidase-conjugated second antibody (1:5000) (Amersham Biosciences). The protein bands were detected by an enhanced chemiluminesence kit (Amersham Biosciences).

The slides were incubated with $3 \%$ bovine serum albumin for $30 \mathrm{~min}$ and then incubated with the anti-AR (1:50), anti-par-4 (1:100), or anti-c-FLIP (1:50) antibody overnight at $4{ }^{\circ} \mathrm{C}$. A streptavidin-biotin peroxidase detection system was used according to the manufacturer's instructions (DAKO, Carpinteria, CA, USA). After being washed with PBS, slides were developed with stable 3,3'-diaminobenzidine and counterstained with $\mathrm{H} \& \mathrm{E}$.

\section{Apoptosis assays}

Flow cytometry was used to assess the sub-G1 DNA population of cells undergoing apoptosis. Cells were released from plates and fixed with 70\% ethanol overnight. Analysis of the sub-G1 DNA content was performed on a flow cytometer (EPICS XL-MCL, Beckman Coulter, Miami, FL, USA) according to the manufacturer's protocol with propidium iodide $(50 \mu \mathrm{g} /$ $\mathrm{ml}$ ) staining. The TUNEL assay was performed with the paraffin sections of LNCaP cells and mouse prostate using the apoptosis detection kit (Promega) according to the manufacturer's instructions. For double staining, slides were first immunostained with anti-c-FLIP antibody with Alexa Fluor 594-conjugated secondary antibody (Molecular Probes, Eugene, OR, USA) and then submitted for the TUNEL assay. The signals were observed under a fluorescent microscope with a red (for c-FLIP staining) or a green (for TUNEL assay) filter. The apoptotic cells were quantified as follows: slides were evaluated under a microscope (Olympus IX71) with a digital camera (Retiga 1300) that was interfaced to a computer with PCI software (Compix Inc., Cranberry Township, PA, USA). The number of epithelial cells of the area of prostate on the image was counted. There were about 300 to 500 cells per area, and three areas were captured on each slide. The fluorescence-labeled cells (apoptotic cells) from the same area of the prostate were captured and counted. Two prostate glands derived from two mice per experimental group were analyzed.

\section{Prostate tissue specimens and pathological evaluation}

For in situ hybridization of par-4 and c-Flip, prostate cancer and normal control tissues were retrieved from archival radical prostatectomy specimens of 44 patients with prostate cancer who had been treated at New York University Medical Centers. In addition, a prostate cancer tissue microarray with hormone naive and resistant cases was constructed and used for immunohistochemistry of c-Flip. Hormone resistant samples were derived from patients who underwent channel transurethral resection (TURP) at least 6 months after surgical prostatectomy. Hormone naive cases were derived from TURP and radical prostatectomy specimens of patients with prostate cancer. Hormone naive TURP specimens were derived from patients who were diagnosed with prostate cancer by TURP, having high grade (Gleason 8 or higher) and volume of disease. A separate group of radical prostatectomy that had previously undergone neoadjuvant therapy (Meyer et al. 1999, Powell et al. 2002) for 3 months prior to radical prostatectomy was also added to the prostate cancer tissue microarray as a neoadjuvant group. Due to the heterogeneous nature of prostate cancer, each case is represented by four $0.6 \mathrm{~mm}$ cores. The study protocol was approved by the New York University institution review board. Clinical samples were fixed in 10\% neutral buffered formalin for 6-24 h and embedded in paraffin. Sections of the tissue $(4 \mu \mathrm{m})$ were cut and mounted on Super-frost Plus adhesion slides (Fisher Scientific Company, Pittsburg, PA, USA) and used for histological analysis, in situ hybridization and immunohistochemistry. The histological features of each individual specimen were confirmed independently by two urology pathologists (P L and J M).

For in situ hybridization, the DNA fragment of the corresponding par-4 and c-Flip cDNA coding region was subcloned into pBluescript II, and the digoxigeninlabeled RNA probes were synthesized by incubation of DNA with T7 or T3 RNA polymerase. After wax removal and rehydration, $4-\mu$ sections of formalin-fixed tissue were hybridized with the digoxigenin-labeled RNA probes, washed and developed by incubation with chromogenic substrate (positive cells stain purple or blue, and negative cells stain colorless or light pink).

Immunohistochemistry was performed on prostate cancer tissue microarray with the anti-c-Flip antibody at 1:50 dilution after antigen retrieval $(20 \mathrm{~min})$ in a microwave in citrus buffer $(\mathrm{pH}$ 6.0). The staining intensity is scored semi-quantitatively as $1+$ for weak staining, 2+ for moderate staining, and 3+ strong staining. The scores were assigned to each core by consensus review of two pathologists.

\section{Results}

\section{Par-4 is a coactivator for AR}

Castration decreased AR expression in androgendependent cells of the prostate gland and, at the same time, induced par-4 expression (Sells et al. 1994). Yeast 
two-hybrid screening with WT1 as bait identified par-4 as a WT1-interacting protein, which modulated the transcriptional activity of WT1 (Johnstone et al. 1996, Richard et al. 2001). On the basis of these observations, we postulated that par-4 might function as a coactivator for $\mathrm{AR}$ to compensate for loss of $\mathrm{AR}$ function due to androgen depletion by castration. To test this hypothesis, transfection experiments were carried out to analyze the effects of par- 4 on transcriptional activation driven by AR. A luciferase reporter (4 xARE-E4-luc) was co-transfected with expression vectors for AR, par-4, or both into PC3 cells in the absence or presence of the synthetic androgen (R1881). As shown in Fig. 1A, AR activated the reporter gene by about sixfold in the presence of R1881, and coexpressed par-4 showed a $5 \cdot 6$-fold enhancement of this activity. Par-4 did not influence reporter gene activity in the absence of R1881, indicating that the enhancing effect of par-4 on AR-dependent gene expression was androgen dependent. Par-4 also enhanced the AR-driven transcription from natural probasin $(-244$ to +12$)$ (Claessens et al. 1996) and the prostate-specific androgen (PSA) enhancer $(-4354$ to -3858$)$ (Schuur et al. 1996) reporter constructs in an androgen-dependent manner (Fig. 1A).

Western blot analysis with anti-AR antibody demonstrated that co-expression of par-4 did not change the protein levels of AR in the transfected cells (Fig. 1B, lane 3 versus lane 2). AR was coimmunoprecipitated with par-4 from the whole-cell extracts made from LNCaP cells grown in the presence (lanes 7-8) and absence (lanes 4-6) of $10 \mathrm{nM} \mathrm{R} 1881$ (Fig. 1B). This finding suggests that par-4 interacted with AR in LNCaP cells. As a negative control, no AR was immunoprecipitated by preimmune serum (lanes 5 and 8). As shown in Fig. $1 \mathrm{~B}$, in vitro-translated par-4 was specifically retained by GST-DBD (lane 13) but not by GST alone (lane 11), GST-NTD (lane 12), or GST-LBD (lanes 14 and 15). These results indicate that par-4 binds specifically to the zinc finger-containing DBD of AR. As the DNA-binding domains of the steroid nuclear receptors are highly conserved, we evaluated the effects of par- 4 on the transcription of reporters containing the same E4 core promoter under the control of estrogen receptor $(\mathrm{ER} \alpha)$, glucocorticoid receptor (GR), progesterone receptor $(\mathrm{PR} \beta)$, and thyroid hormone receptor (TR $\alpha)$. Par-4 also enhanced ER $\alpha$-, GR-, and PR $\beta$-driven gene expression; in contrast, par- 4 showed no effect on TR $\alpha$-mediated transcription (data not shown). Hence, par-4 enhanced the steroid hormone receptor-dependent transcription in vivo.

\section{Par-4 exists as an octamer in HeLa cells}

The 6 His-tagged par-4 was expressed and purified from bacteria and had an apparent molecular mass of $44 \mathrm{kDa}$, as determined by SDS-PAGE (Fig. 2A). It has been shown that par-4 interacts with itself through the C-terminal leucine zipper domain (Dutta et al. 2001). To characterize the molecular properties of par-4 in vivo, we generated a stable cell line expressing FLAG epitopetagged par-4. Immunopurification of f:par-4 from nuclear extracts made from f:par-4 cells revealed the association of a 44-kDa polypeptide with f:par-4 (Fig. 2B, lanes 3 and 4, indicated by a white arrow). The specific association of this polypeptide with f:par-4 is further demonstrated by the failure of similar-sized polypeptides in extracts from control cells (not expressing f:par-4) to bind to the affinity matrix (lane 2). Close examination of the silver-stained gel did not reveal any other polypeptides associated with f:par-4 (Fig. 2B, lane 3 versus lane 2). The band (Fig. 2B, lanes 3 and 4, indicated by a star on the right) was a degraded product of par-4 because it was recognized by anti-par-4 antibody (Fig. 2D, indicated by a star on the left). Western blot analysis with anti-par-4 antibody demonstrated that the f:par-4 associated 44-kDa polypeptide in the immunoprecipitate was the endogenous par-4 (Fig. 2C, lane 6). The f:par-4 also associated with endogenous par-4 in the cytoplasm (Fig. 2C, lane 4).

To determine the native size of par-4, the immunoprecipitate was analyzed by gel-filtration chromatography on a Sepharose 6 column. Western blot analysis of the fractions revealed that f:par-4 and endogenous par-4 were coeluted, and most of the FLAG-tagged and nontagged par-4 was coeluted in fractions \#16 to \#18, corresponding to a native size of about $360 \mathrm{kDa}$ (Fig. 2D). As this is roughly eight times the molecular mass of par-4 determined by SDS-PAGE, these findings suggest that par-4 is an octamer in HeLa cells.

\section{The $\mathrm{N}$-terminal peptide of par-4 functions as a dominant negative}

The par-4 protein is highly conserved during evolution throughout the entire coding region. The N-terminal (amino acid residues 1-36), middle (amino acid residues 135-201), and C-terminal (269-342) parts of par-4 are the most conserved (Fig. 3B). The C-terminal part contains a leucine zipper (amino acid residues 301-342). On the basis of these structural features, we generated a set of deletions (Fig. 3B). The deletion variants of par-4 were subcloned into the mammalian expression vector (pcDNA3 1) and transiently transfected into PC3 cells (Fig. 3A). Western blot analysis revealed that expression of these truncation constructs occurred at similar levels (data not shown). Deletion of up to the first 145 amino acid residues from the $\mathrm{N}$-terminal did not apparently affect the ability to enhance AR-transactivation. However, further deletion from the N-terminal completely abolished the ability of par-4 to increase AR-dependent transcription. The deletion of the C-terminal up to amino acid residue 201 did not affect 
A
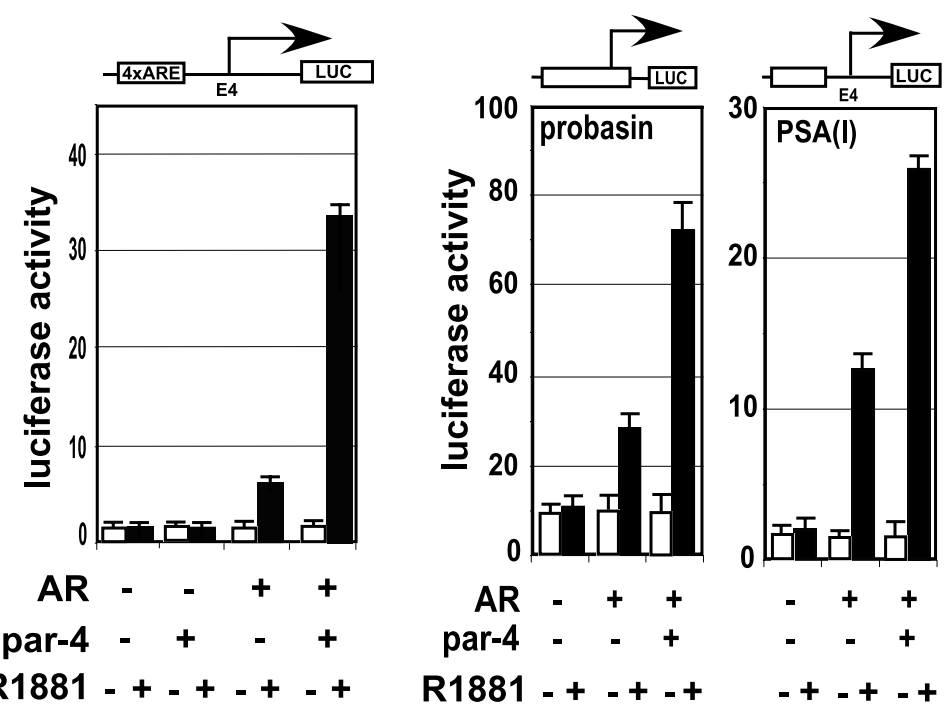

B

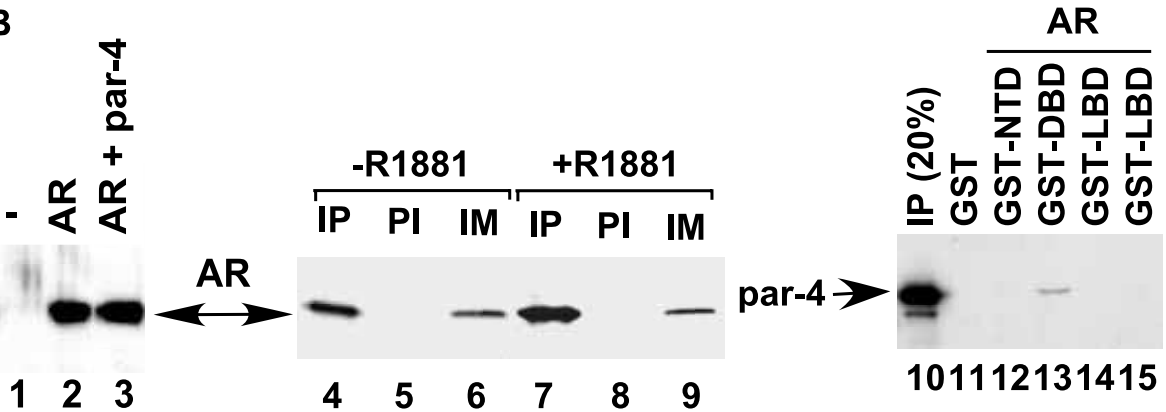

C

Qn

Gn

DBD Hinge

LBD

AR
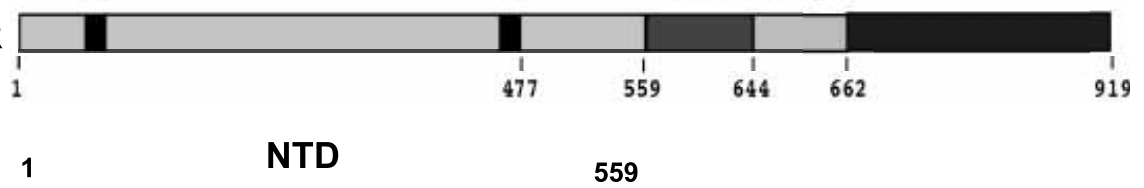

559

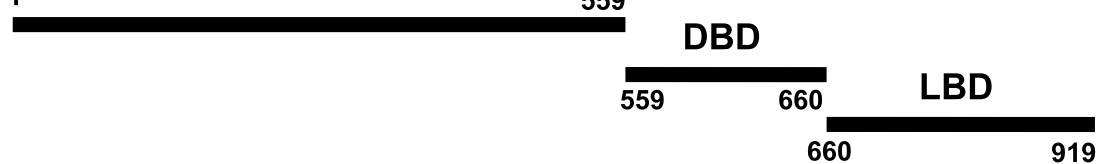

Figure 1 Par-4 increased AR-driven gene expression. (A) PC3 cells were transfected with 25 fmoles 4 xARE-, probasin-, or PSA(I)-luc reporter plasmid, 1.5 fmoles pcDNA-AR, and 30 fmoles pcDNA-par-4 expression plasmid. Cells were grown in the absence or presence of $10 \mathrm{nM} \mathrm{R} 1881$ for $36 \mathrm{~h}$ after transfection and then harvested for luciferase activity assays. LUC, luciferase.

(B) Par-4 interacted with the DBD of AR. Western blot analysis of whole-cell extracts (10 $\mu$ l) made from PC3 cells (lane 1) and PC3 cells transiently transfected with AR (lane 2) or AR plus par-4 (lane 3) using anti-AR antibody is shown. Par-4 interacted with AR in vivo. The whole-cell extracts were made from LNCaP cells grown in the presence (lanes 7-9) or absence (lanes 4-6) of 10 nM R1881. Whole-cell extracts, $0.5 \mathrm{ml}(0.2 \mathrm{mg}$ protein), were mixed with $10 \mu \mathrm{l}$ of the anti-par-4 serum (IM) (lanes 6 and 9$)$ or preimmune serum (PI) (lanes 5 and 8). The mixtures were incubated at $4{ }^{\circ} \mathrm{C}$ for $1 \mathrm{~h}$, and then $10 \mu \mathrm{l}$ protein $\mathrm{A}$ Sepharose beads were added. The mixtures were incubated for $1 \mathrm{~h}$ and, after being washed, the beads were submitted for Western blot analysis with anti-AR antibody. Lanes 4 and 7 are the whole-cell extracts $(5 \mu \mathrm{l})$ used for the immunoprecipitation (IP). AR interacted with the DBD of AR. Glutathione agarose beads $(10 \mu \mathrm{l})$ containing $1 \mu \mathrm{g}$ GST (lane 11), GST-NTD (lane 12), GST-DBD (lane 13), or GST-LBD (lanes 14 and 15) of AR were incubated with ${ }^{35}$ S-labeled par-4 in the absence (lanes 11-14) or presence (lane 15) of 100 nM $\mathrm{R} 1881$ for $2 \mathrm{~h}$ at $4{ }^{\circ} \mathrm{C}$. After being washed, the beads were boiled with SDS sample buffer and subjected to SDS-PAGE, followed by autoradiography. Lane 10 is $20 \%$ input (IP) for the binding assay. (C) Diagram of the AR domains used for the interaction assay in B. Qn and Gn represent glutamine and glycine stretches respectively. 
A
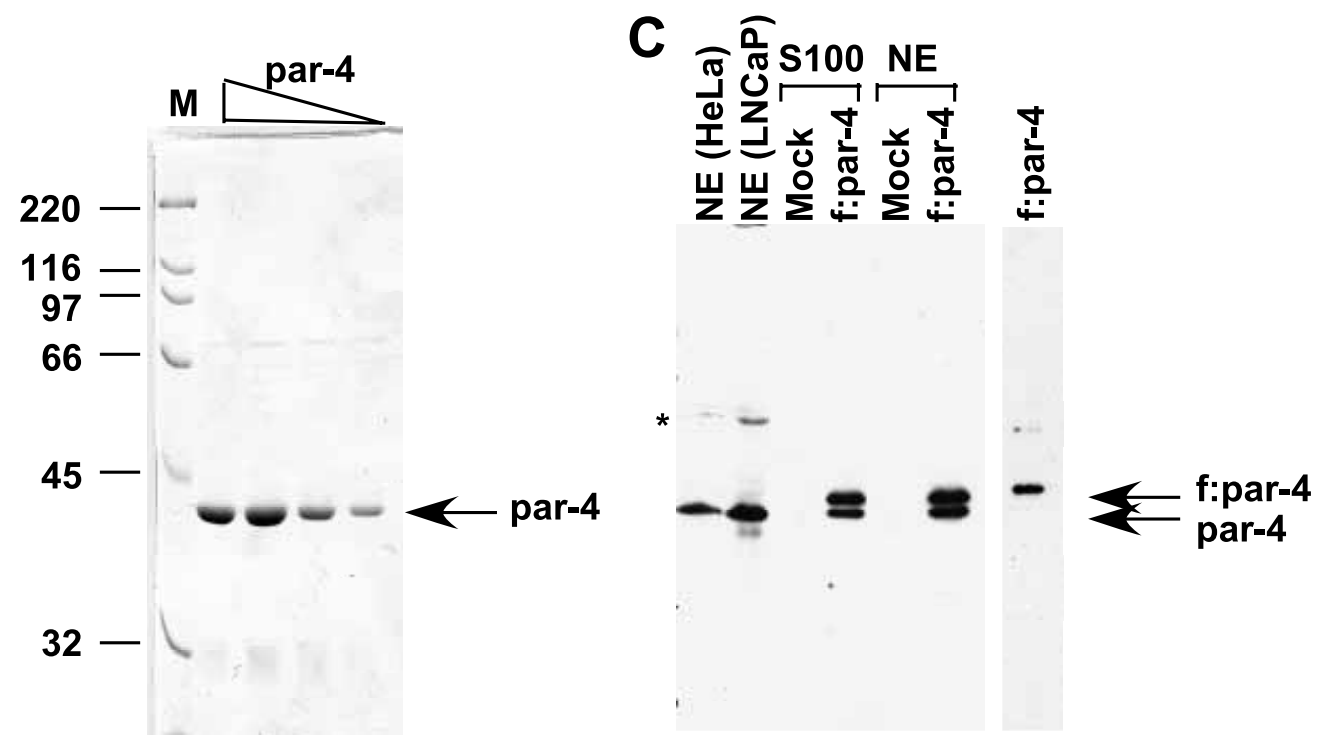

$\begin{array}{lllll}1 & 2 & 3 & 4 & 5\end{array}$

$\begin{array}{lllllll}1 & 2 & 3 & 4 & 5 & 6 & 7\end{array}$

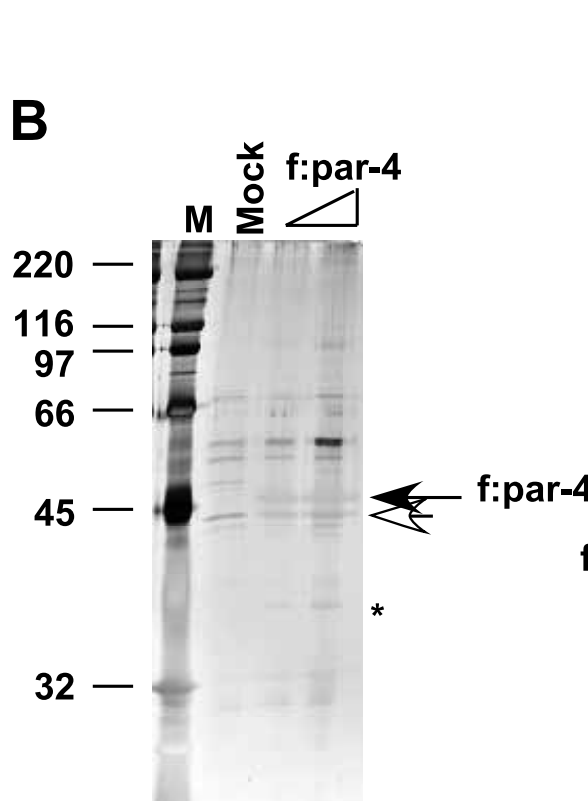

$123 \quad 3 \quad 4$
D

\section{Fraction \# IP 11121314151617181920212223}

\section{$360 \mathrm{kDa}$}

Figure 2 Par-4 forms an octamer in HeLa cells. (A) The 6 His-tagged par-4 was expressed and purified from bacteria. One hundred (lane 5), 200 (lane 4), or 400 (lanes 2 and 3) ng purified recombinant par-4 were analyzed by 10\% SDS-PAGE with Coomassie Blue R250 staining. (B) SDS-PAGE analysis of the purified f:par-4-containing complex. Immunoprecipitation was performed with nuclear extracts made from a stably transfected, FLAG-tagged, par-4-expressing cell line. Bands corresponding to FLAG-tagged par-4 and polypeptides specifically associated with par- 4 are indicated by the arrows on the right. Lane 2 (Mock) shows

immunoprecipitate obtained from the control cell line. Lane 1 shows standard molecular weight markers (Bio-Rad Laboratories, Inc., Hercules, CA, USA). (C) Western blot analysis of the f:par-4-complexes isolated from cytoplasm (lanes 3 and 4) or nuclear extract (lanes 5, 6, and 7) of the f:par-4 (lanes 4, 6, and 7) or control (lanes 3 and 5) cell lines with anti-par-4 (lanes 1-6) and anti-FLAG (lane 7) antibodies. The arrows indicate the bands corresponding to f:par-4 and par-4. Lanes 1 and 2 contain $5 \mu$ nuclear extract (NE) made from HeLa or LNCaP cells as indicated. The star on the left indicates a protein that non-specifically cross-reacted with the c-FLIP antibody. (D) Fifty microliters of the FLAG-eluted immunoprecipitate from 2.7 mg nuclear extract made from the f:par-4 cell line were chromatographed on a Superose 6 column in BC300-0.1 NP-40. Ten microliters of each fraction were analyzed by immunoblot with anti-par-4 antibody. Arrows at the top indicate the elution peaks of blue Dextran (2000 kDa), ferritin (440 kDa), aldolase $(158 \mathrm{kDa})$, bovine serum albumin $(67 \mathrm{kDa})$, and ovalbumin $(43 \mathrm{kDa})$. Arrows on the left indicate bands corresponding to par-4 and f:par-4. 


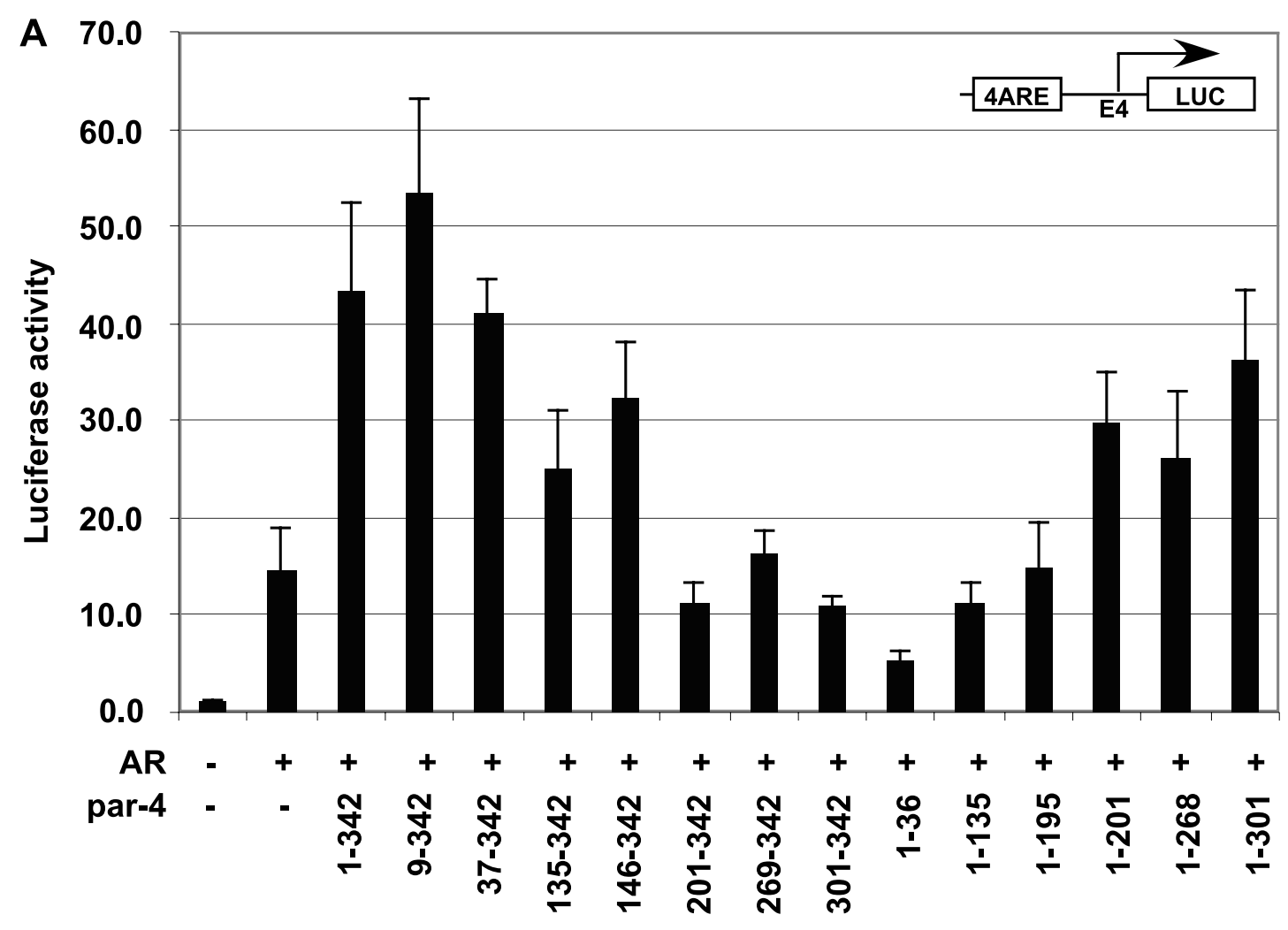

B

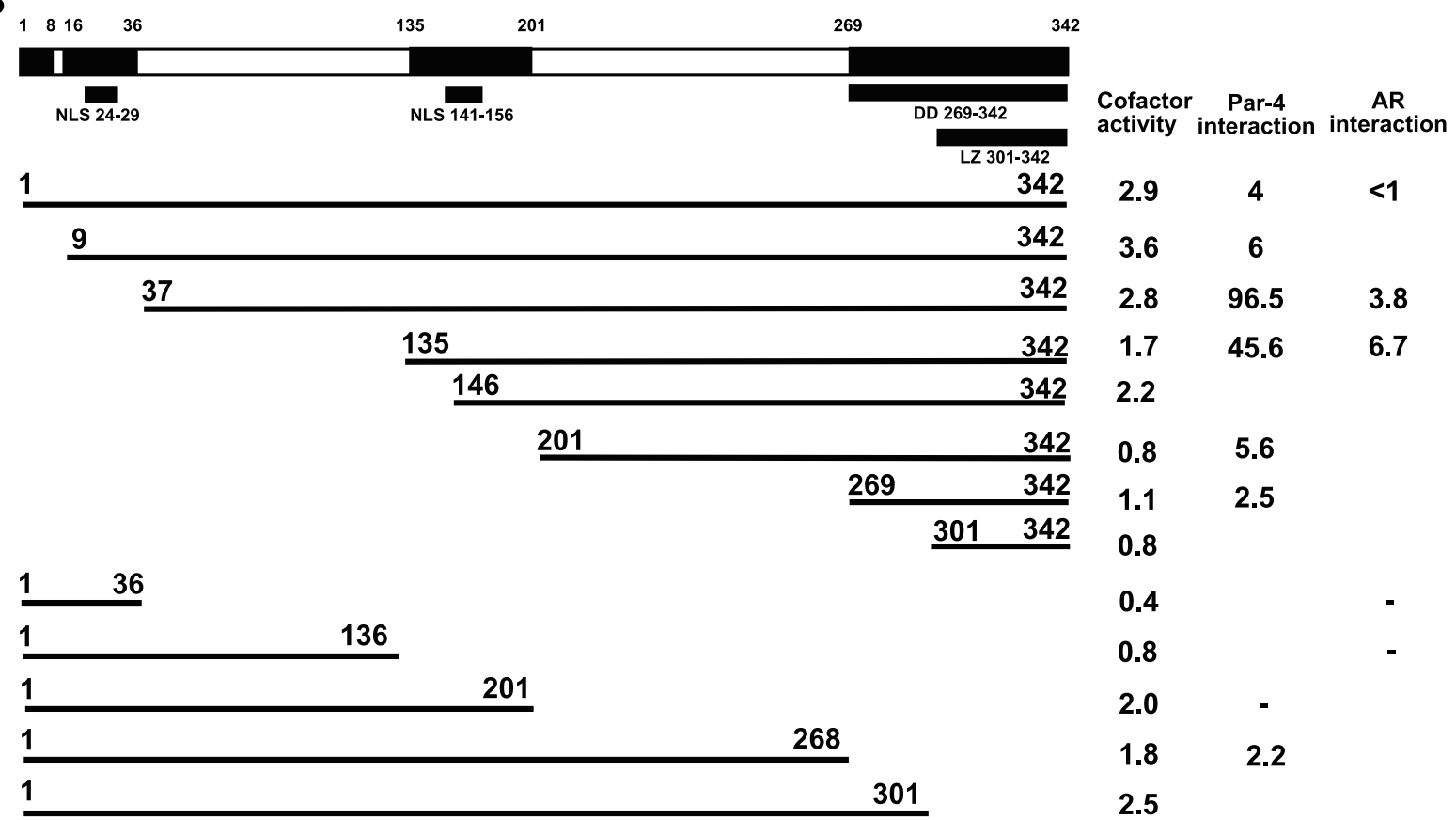

Figure 3 Functional analysis of the par-4 variants. (A) PC3 cells were transfected with 25 fmoles of the reporter plasmid (p4 xARE-E4-luc), 30 fmoles of various par-4 truncations, and 1.5 fmoles pcDNA-AR. (B) Schematic diagram of various full-length or truncated par- 4 proteins. The highly conserved regions are represented by black boxes. The numbers at the left indicate the fold-activation of AR-driven transcription by par-4 variants (cofactor activity) or percentage of ${ }^{35} \mathrm{~S}$-labeled input proteins retained on GST-par-4 beads (par-4 interaction) or GST-AR DBD beads (AR interaction). 
par-4-induced luciferase activity, but further deletion resulted in complete loss of par-4-induced luciferase activity. Thus, the central domain (amino acid residues 146-201) of par-4 is important for the enhancement of AR-dependent transactivation. The N-terminal peptide (amino acid residues 1-36) of par-4 functioned as a dominant negative, indicating that endogenous par-4 contributed to the observed AR-dependent transcription in PC3 cells.

\section{An intermolecular interaction exists in the par-4 molecule}

Next, using an in vitro transcription and translation system, we produced a series of $\left[{ }^{35} \mathrm{~S}\right]$ methionine-labeled par-4 deletion variants. These variants were incubated with GST-par-4 immobilized on glutathione Sepharose beads. After incubation, the beads were washed extensively. The bound ${ }^{35} \mathrm{~S}-$ par- 4 variants were then analyzed by SDS-PAGE and autoradiography (Fig. 4A). As a negative control for nonspecific binding, GSTglutathione Sepharose beads were incubated with ${ }^{35} \mathrm{~S}-$ par- 4 variants at the same time under the same conditions. The full-length ${ }^{35} \mathrm{~S}-$ par-4 (Fig. 4A, lanes 1-3) failed to associate with GST-beads (lane 2) but was retained on the par-4-beads (lane 3). ${ }^{35} \mathrm{~S}$-par-4 variants lacking the C-terminal 74-amino acid residues weakly interacted with par-4 beads (Fig. 4A, lanes 22-24), indicating a requirement of this region (leucine zipper domain) for binding. In contrast, all ${ }^{35} \mathrm{~S}-$ par-4 deletion variants possessing the C-terminal 141 amino acid residues bound to GST-par-4 beads (Fig. 4A, lanes 6, 9, 12 , and 15), revealing that this region is necessary and sufficient for interaction. However, the ${ }^{35} \mathrm{~S}-$ par-4 variant with 36 amino acid residues deleted from the N-terminal bound to GST-par-4 beads with much higher affinity (Fig. 4A, lane 9 versus lane 3), indicating that the N-terminal 36 amino acid residues inhibited the interaction between par- 4 and itself. It was interesting that ${ }^{35}$ S-labeled par-4 (269-342) was retained on the GSTpar-4 (1-36) beads (Fig. 4B, lanes 1-3), suggesting that there is an intermolecular interaction within the par-4 molecule. This intermolecular interaction required the intact dimerization domain (amino acid residues 269342) (Fig. 4B, lanes 4-9). Under the same conditions, the $\mathrm{N}$-terminal part (amino acid residues 1-136) did not show any intermolecular interaction (Fig. 4B, lanes 19-21).

A similar analysis was performed on heteromolecular interactions between par-4 and AR. The results are summarized in Fig. 3B. Similarly, the intermolecular interaction of par- 4 inhibited the interaction of par-4 with AR. These results suggest that there is an intermolecular interaction within the par-4 molecule and that this interaction inhibits the homomolecular and heteromolecular interactions within par- 4 and between par-4 and AR.

\section{Par-4 enhanced AR-ARE interaction}

After demonstrating that the DBD of AR mediates the interactions of AR with par-4, we investigated whether par-4 affects AR-ARE interaction. A DNA probe containing the ARE derived from the PSA promoter $(-152$ to -174$)$ (Cleutjens et al. 1996) was used for a gel shift assay (Fig. 5). Par-4 (1-301) (Fig. 5A, lane 2) and the DBD of AR (amino acid residues 537 to 644) (Liu et al. 2003) were expressed as a 6 His-tagged fusion protein and purified through an NTA $\mathrm{Ni}^{2+}$ agarose affinity column. The recombinant DBD shifted the probe (Fig. 5B, lanes 2 and 7), and addition of the recombinant par-4 (1-301) (lanes 3-5), not BSA (lanes 8-10), enhanced DBD-ARE complex formation in a dose-dependent manner. Mutations on the conserved nucleotides in the ARE abolished DBD-ARE complex formation (lanes 12-14), indicating that the par-4mediated enhancement of the AR-ARE interaction was dependent on functional ARE. The addition of par-4 did not change the migration of DBD-ARE, suggesting that par-4 was not in the DBD-ARE complex in the gel. This may reflect instability of the tentative complex of par-4-AR-ARE in the electrophoresis assay. A similar phenomenon was observed in another study when the thyroid hormone receptor/TR-associated proein (TR/ TRAP) and TATA-box binding protein/TBP-associated factors (TBP/TAFs) complexes interacted with their cognate DNA elements in a gel shift assay (Fondell et al. 1996). Similarly, par-4 (1-301) also dramatically increased the density of the AR-ARE band (up to 10-fold) (Fig. 5C, lanes 3-5 versus lane 2). Under the same conditions, we did not observe any interaction between par-4 and the DNA probe (data not shown). The same assay was performed with the full-length par-4 expressed in and purified from bacteria and results were not conclusive due the aggregation/polymerization of par-4 protein (data not shown). These results indicate that enhanced AR transactivation by par-4 may occur through the increased binding affinity of AR to the ARE in the presence of par-4.

\section{Expression of $\mathrm{N}$-terminal peptide of par-4 induced apoptosis in LNCaP cells}

We tried to fuse the FLAG epitope at the $\mathrm{N}$ - or $\mathrm{C}$-terminal end of the $\mathrm{N}$-terminal amino acid residues 1-36 (N-terminal peptide) of par-4 to detect its expression in cells, but the FLAG epitope tag abolished the dominant negative function of the N-terminal peptide (data not shown). To overcome this obstacle, we constructed an expression vector (Fig. 6A). This vector included an internal ribosomal entry site (IRES) and expressed one mRNA that encodes the $\mathrm{N}$-terminal peptide of par-4 and EGFP. Therefore, the presence of EGFP indicated that the N-terminal peptide of par-4 
A

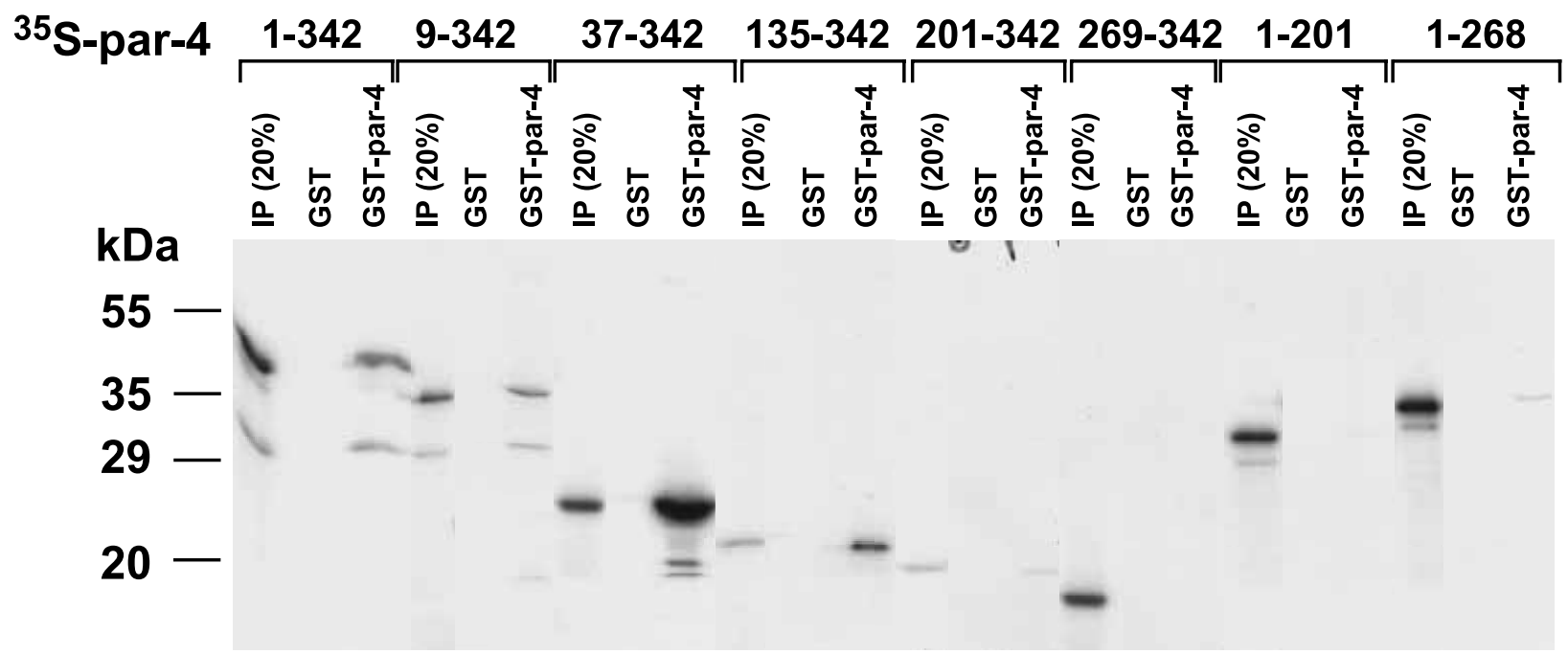

$\begin{array}{llllllllllllllllllllllll}1 & 2 & 3 & 4 & 5 & 6 & 7 & 8 & 9 & 10 & 11 & 12 & 13 & 14 & 15 & 16 & 17 & 18 & 19 & 20 & 21 & 22 & 23 & 24\end{array}$

B

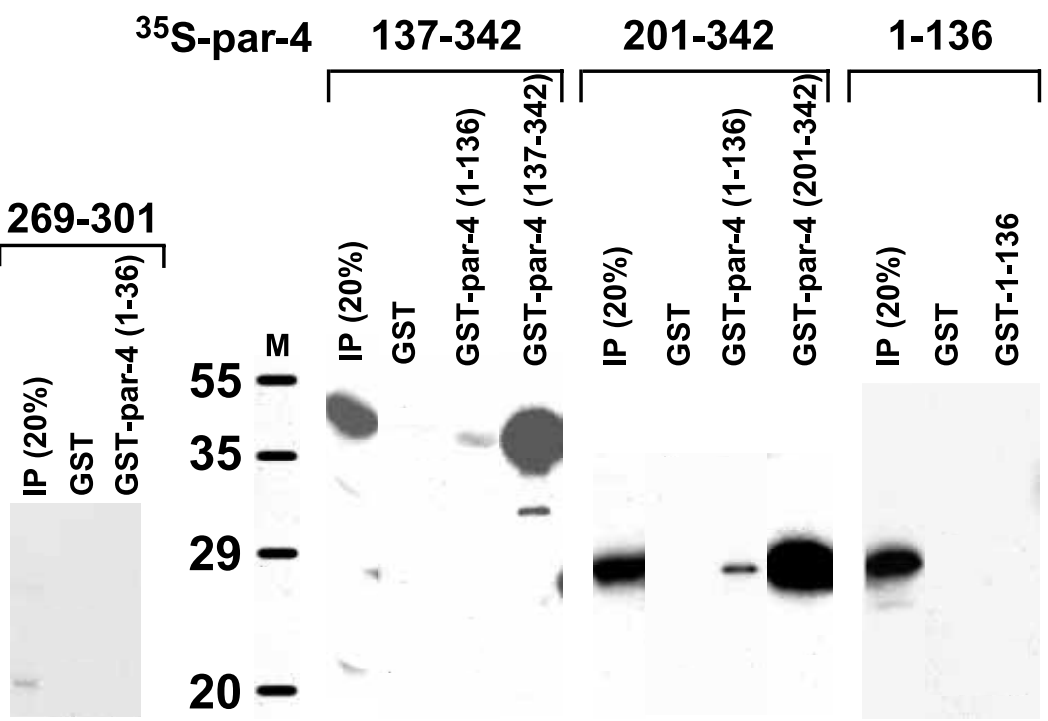

${ }^{35}$ S-par-4 269-342 301-342 269-301

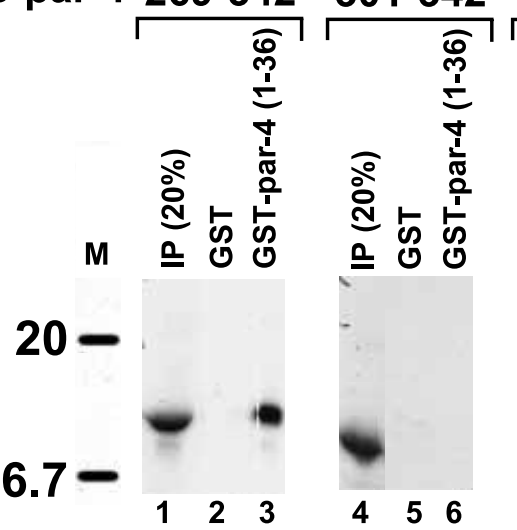

789

$\begin{array}{llllllllllll}10 & 11 & 12 & 13 & 14 & 15 & 16 & 17 & 18 & 19 & 20 & 21\end{array}$

Figure 4 GST-fusion protein pull-down assay reveals the intermolecular interaction of par-4. (A) The full-length or deletion variants of par-4 were labeled with [ ${ }^{35}$ S] methionine. The labeled proteins were incubated with GST (lanes 2, 5, 8, 11, 14, 17, 20, and 23) or GST-par-4 (lanes 3, 6, 9, 12, 15, 18, 21, and 24) beads. After extensive washing, the bound proteins were analyzed by SDS-PAGE and autoradiography. The $20 \%$ of the labeled proteins (IP) used for the interaction assay were loaded on the same gel (lanes 1, 4, 7, 10, 13, 16, 19, and 22). (B) The labeled variants of par-4 were incubated with GST (lanes 2, 5, 8, 12, 16, and 20), GST-par-4 (1-36) (lanes 3, 6, and 9), GST-par-4 (1-136) (lanes 13, 17, and 21), GST-par-4 (137-342) (lane 14), or GST-par-4 (201-342) (lane 18) beads, and after extensive washing, the bound proteins were analyzed by SDS-PAGE and autoradiography. The $20 \%$ of the labeled proteins (IP) used for the interaction assay were loaded on the same gel (lanes $1,4,7$, 11,15 , and 19).

was expressed (Fig. 6B). LNCaP cells were transfected with this construct, and the stable cell lines were obtained by G418 selection. One control line (transfected with the empty vector and expressing EGFP only)
(Fig. 6B, panel a) and two positive lines (\#1 and \#6) (panels $\mathrm{b}$ and $\mathrm{c}$ ) with the moderate EGFP signals were chosen for further analysis. We evaluated the effect of $\mathrm{N}$-terminal peptide expression on apoptosis in LNCaP 
C par-4 (1-301) BSA

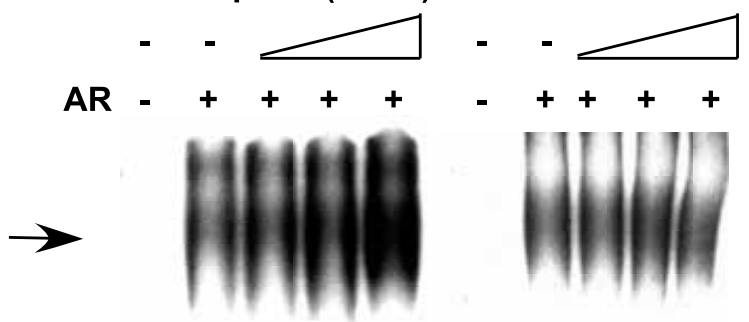

A
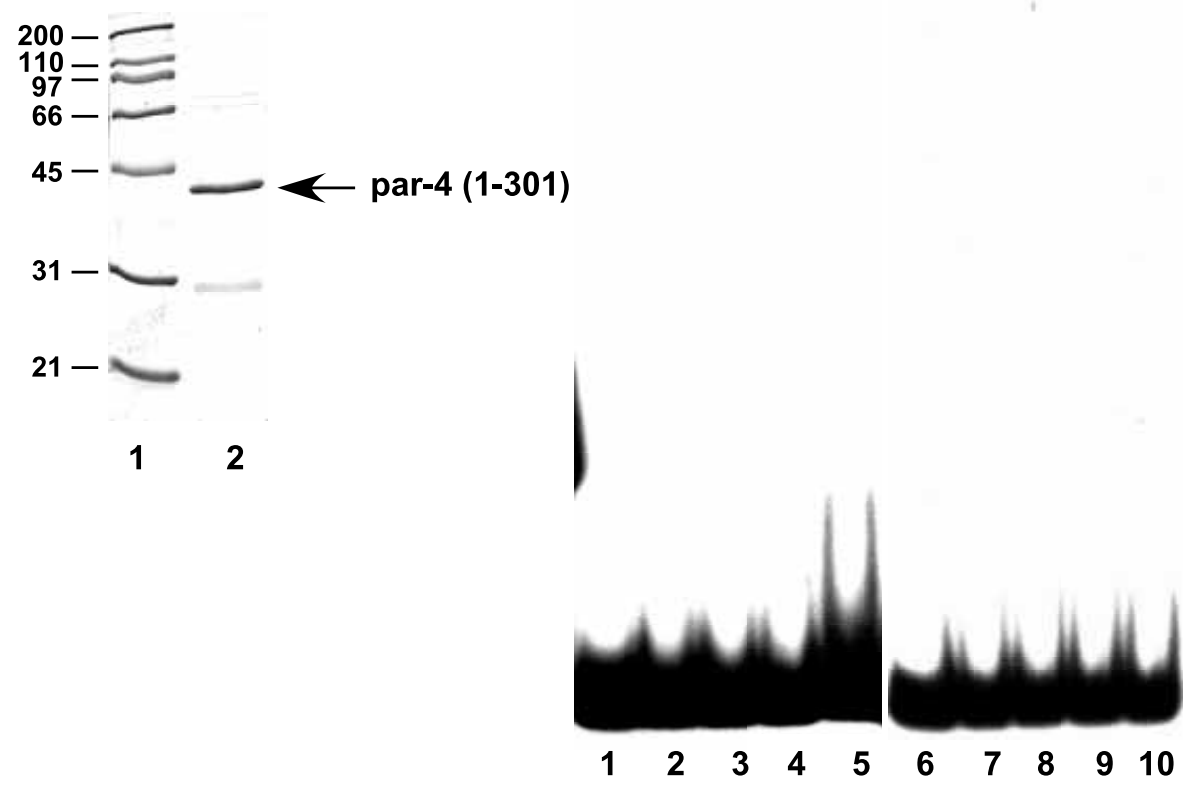

B

par-4 (1-301)

BSA

par-4 (1-301)
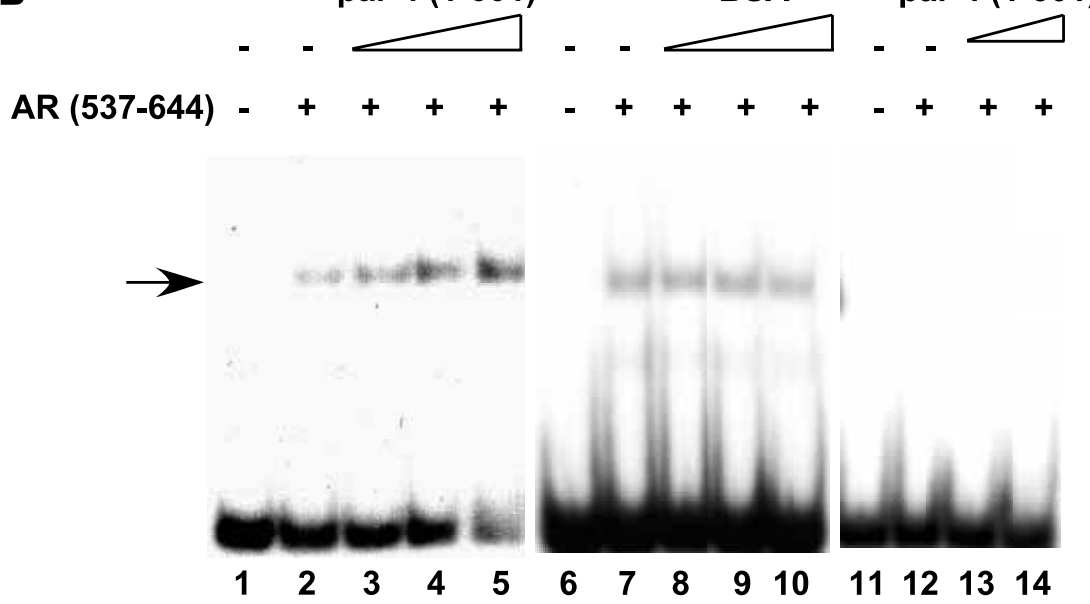

Figure 5 Par-4 enhanced AR interaction with the ARE. (A) SDS-PAGE analysis of the recombinant par-4 (1-301). Five hundred nanograms par-4 (1-301) (lane 2) were expressed, purified from bacteria, and subjected to SDS-PAGE with Coomassie Blue R250 staining. Lane 1 shows standard protein markers (Bio-Rad). (B) The gel shift assay was performed using a DNA probe containing the wild-type (lanes 1-10) or mutant (lanes 11-14) ARE and $1 \mathrm{ng}$ of the recombinant AR (537-644) (Liu et al. 2003). Ten nanograms (lanes 3 and 8), $30 \mathrm{ng}$ (lanes 4, 9 and 13), or $90 \mathrm{ng}$ (lanes 5, 10, and 14) of the recombinant par-4 (1-301) or BSA were included in the binding reactions as indicated. (C) The gel shift assay was performed using a DNA probe containing the PSA ARE and $80 \mathrm{ng}$ of the full-length AR expressed and purified from Sf9 cells (Yu et al. 1994). Ten nanograms (lanes 3 and 8 ), $30 \mathrm{ng}$ (lanes 4 and 9 ), or $90 \mathrm{ng}$ (lanes 5 and 10) of the recombinant par-4 (1-301) or BSA were included in the binding reactions as indicated. 
A

\begin{tabular}{|c|c|c|c|c|c|c|}
\hline LTR & & IRES & EGFP & SV40 & NEO & LTR \\
\hline LTR & par-4 (1-36) & IRES & EGFP & SV40 & NEO & LTR \\
\hline
\end{tabular}

B
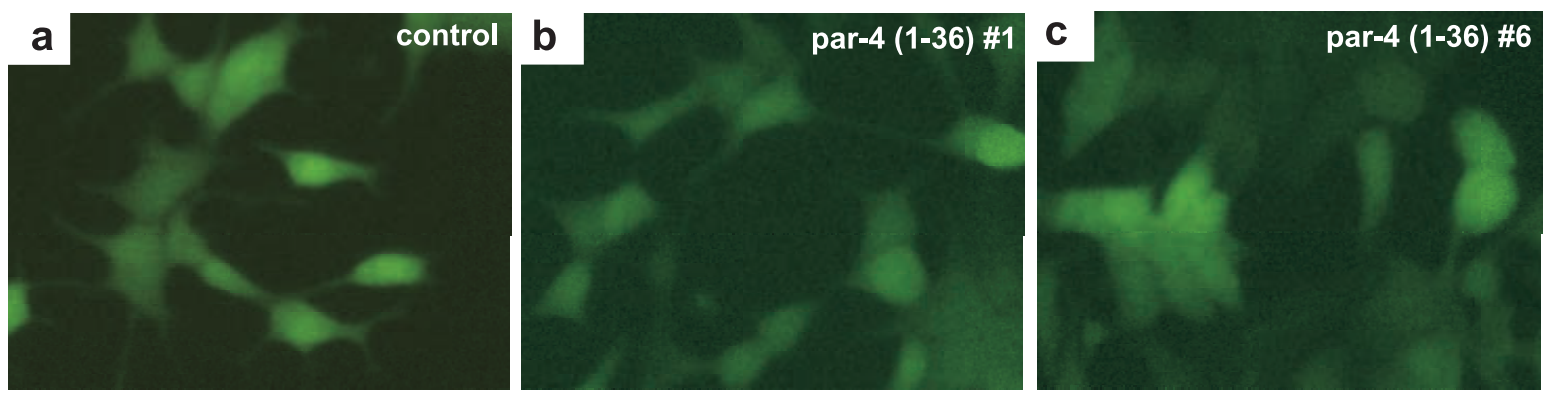

C

Cell Cycle
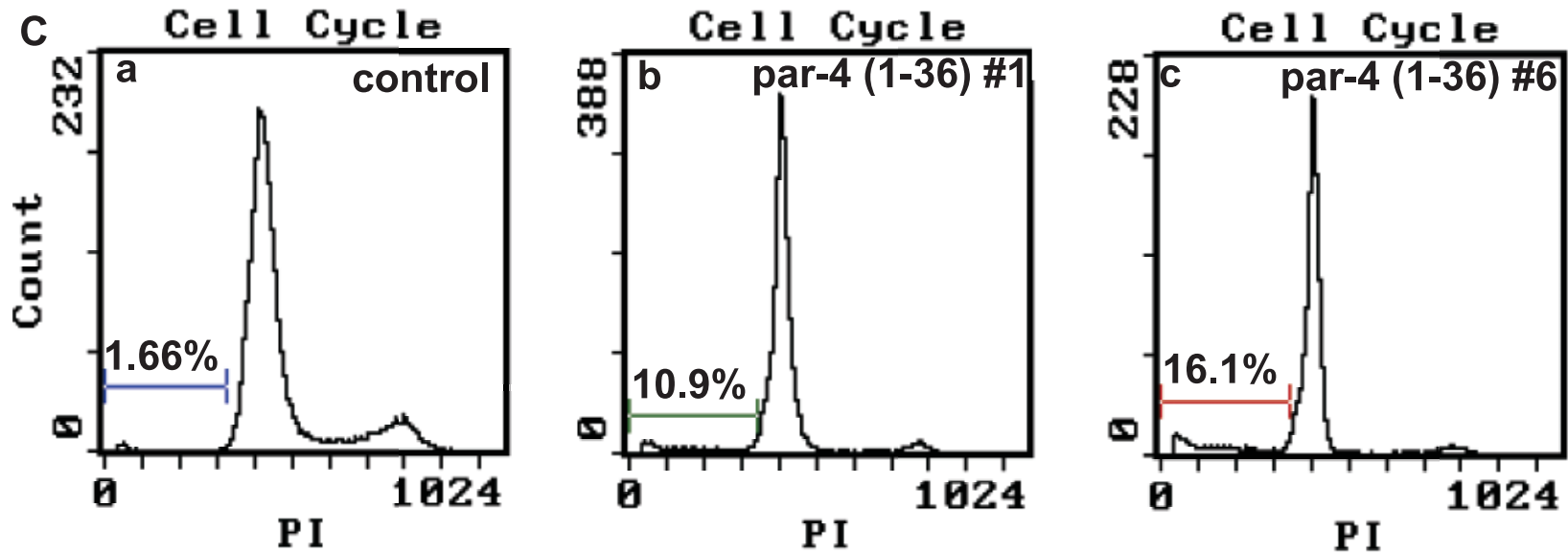

D a

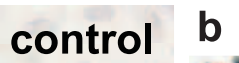

par-4 (1-36) \#1 $\mathrm{c}$
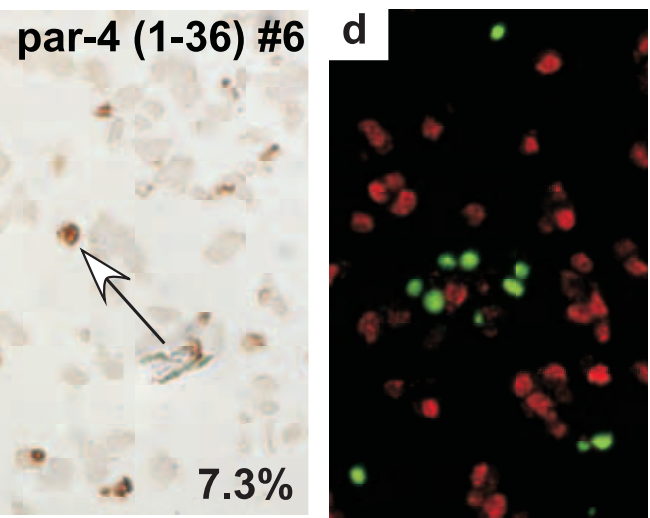

Figure 6 Expression of the N-terminal peptide of par-4 led to apoptosis in LNCaP cells. (A) Schematic diagram of constructs used to express EGFP and the N-terminal peptide of par-4. (B) EGFP expression in LNCAP stable cell lines was observed with a fluorescent microscope. (C) Flow cytometric analysis of sub-G1 content of cells; the percentage of cells that were undergoing apoptosis is indicated. PI, propidium iodide. (D) Apoptosis was measured by the TUNEL assay. Apoptotic cells are stained brown (indicated by white arrows). The percentage of cells undergoing apoptosis is indicated. The left panel (d) shows the double staining of the same slide with anti-c-FLIP (red) and TUNEL (green). LTR, Long terminal repeat; NEO, neomycin. 
A

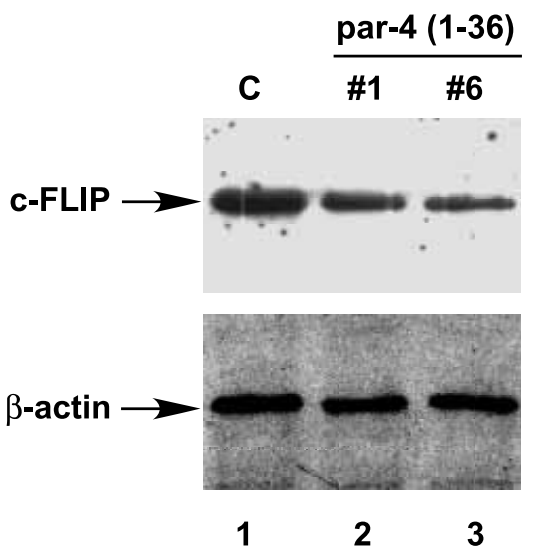

B
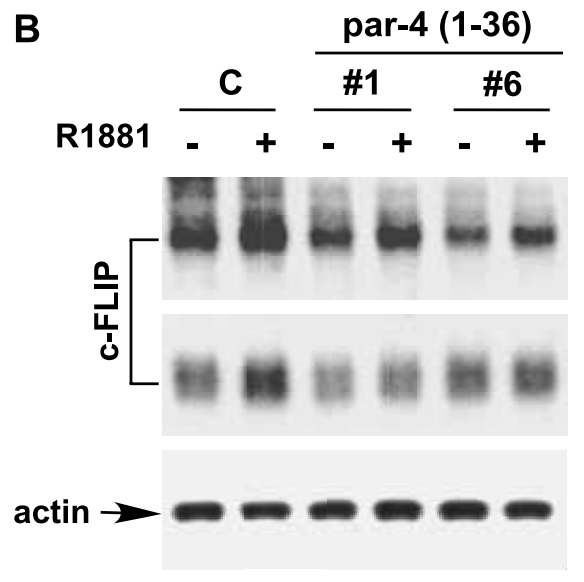

1

456
D

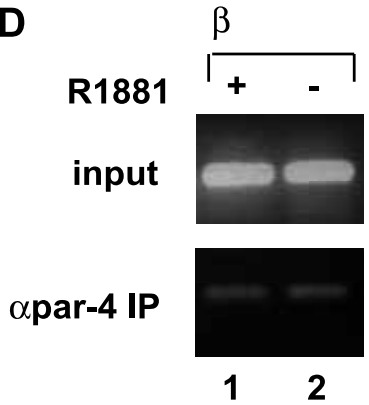

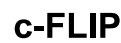
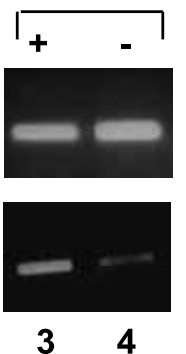

C

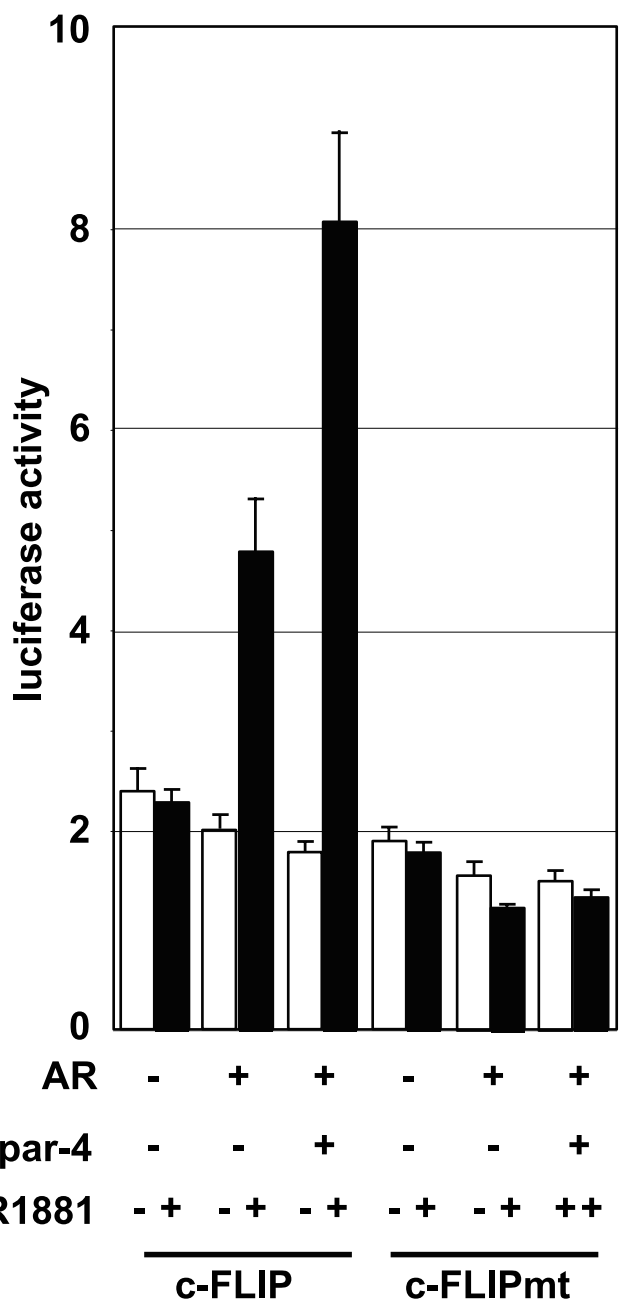

Figure 7 Par-4 directly targets the c-FLIP gene. (A) Western blot analysis of the control (lane 1) and par-4 (1-36) \#1 (lane 2) and \#6 (lane 3) LNCaP cell lines with anti-c-FLIP (top panel) or anti- $\beta$-actin (bottom panel) antibodies is shown. (B) Northern blot analysis of c-FLIP mRNA expression in control (lanes 1 and 2) and par-4 (1-36) (lanes 3-6) LNCaP cell lines is shown. Cells were grown in the absence (lanes 1, 3, and 5) or presence (lanes 2, 4, and 6) of $10 \mathrm{nM}$ R1881 for 2 days. mRNA was isolated, fractionated by electrophoresis, and transferred to a Hybond $\mathrm{N}^{+}$membrane. The membrane was hybridized with c-FLIP probe (top two panels) or $\beta$-actin probe (bottom panel). (C) Activation of the c-FLIP promoter by par-4. PC3 cells were transfected with 25 fmoles of the wild-type c-FLIP report promoter $(-48$ to +156$)$ or the ARE1,2-mutated (C61T, G67A, C95T, G101A) c-FLIP reporter promoter (c-FLIPmt) with or without 15 fmoles pcDNA-AR and 30 fmoles pcDNA-par-4. The transfected cells were grown in the presence or absence of $10 \mathrm{nM}$ R1881 for $36 \mathrm{~h}$ and submitted for dual-luciferase assay. (D) Chromatin immunoprecipitation assay to identify the c-FLIP gene as a direct target gene of par-4. LNCaP cells were grown in the absence (lanes 2 and 4 ) or presence (lanes 1 and 3) of $10 \mathrm{nM}$ R1881. Immunoprecipitation (IP) was performed with antigen-purified anti-par-4 antibodies. The cross-links in the immunoprecipitated protein-DNA complexes were reversed, and the resulting DNA was amplified by a PCR with two specific primers derived from promoter regions of $\beta$-actin (lanes 1 and 2) or c-FLIP (lanes 3 and 4). The same set of PCRs was performed with chromatin DNA used for immunoprecipitation (top panel). 
A

2 days

7 days

15 days

30 days
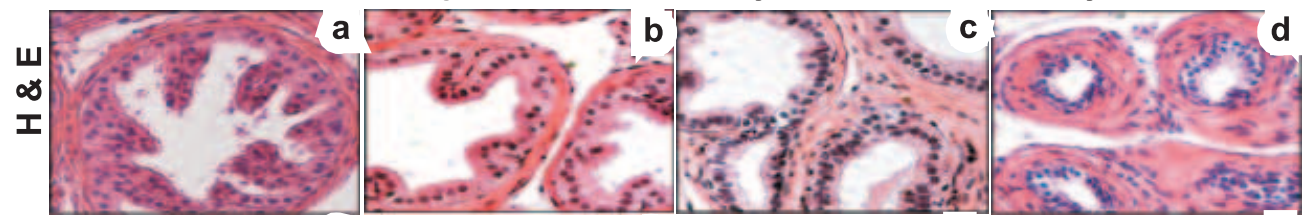

$\left.\frac{1}{4}\right)^{2}$
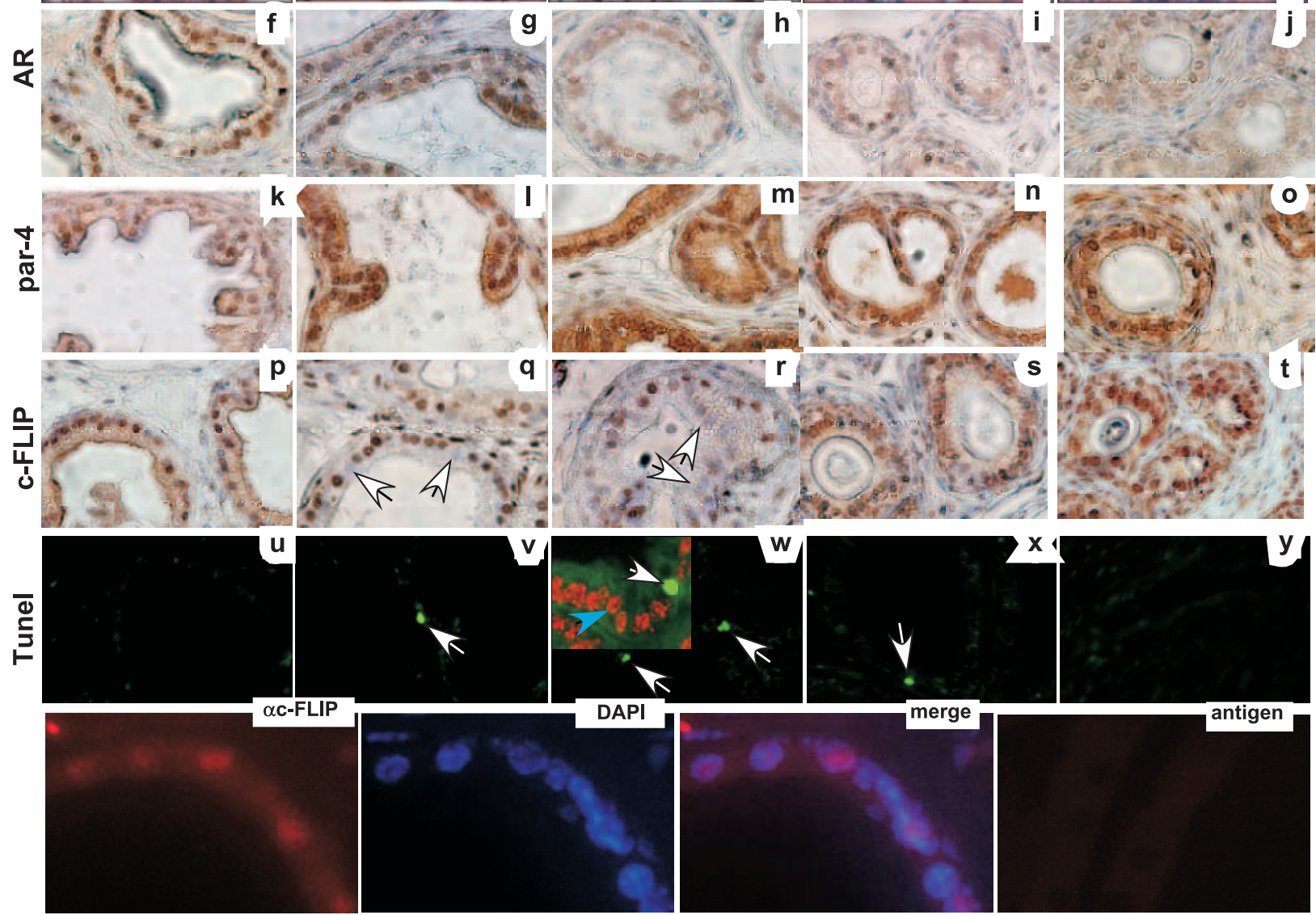

B
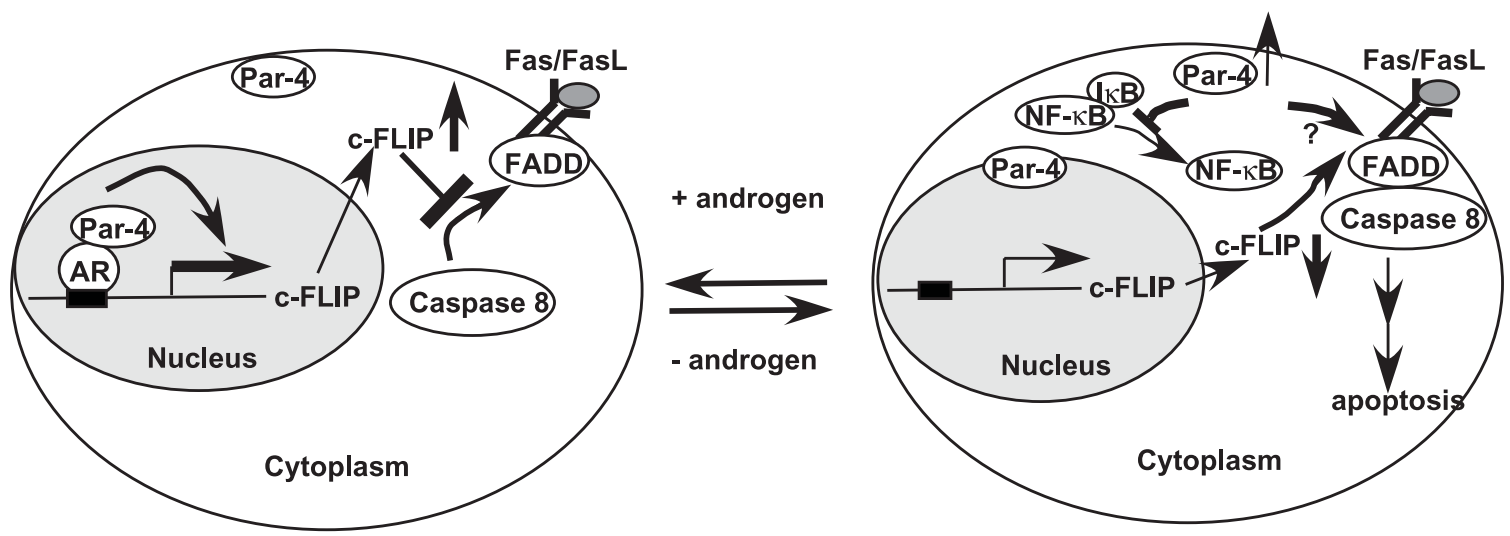
cells by flow cytometry. A low percentage $(1 \cdot 6 \%)$ of EGFP-LNCaP cells were apoptotic (Fig. 6C, panel a), whereas par-4(1-36)-LNCaP cells showed much higher percentages (\#1: $10 \cdot 9 \%$ and \#6: $16 \cdot 1 \%$ ) of cell death (Fig. 6C, panels b and c). Similarly, the TUNEL assay (Fig. 6D) revealed that very few apoptotic cells $(0 \cdot 5 \pm 0 \cdot 3 \%)$ were detectable in the EGRP-LNCaP cell line (panel a). However, more apoptotic cells (\#1: $6 \cdot 5 \pm 2 \cdot 2 \%$ and $\# 6$ : $7 \cdot 3 \pm 2 \cdot 6 \%$ ) were found in par-4(1-36)-LNCaP cell lines (panels b and c, indicated by white arrows). These results suggest that expression of the N-terminal peptide of par-4 induced apoptosis in LNCaP cells.

\section{Par-4 directly targets the c-FLIP gene}

In a previous study, par-4-induced apoptosis of the AR-negative prostate cancer cells (PC3, DU-145, and TSU-Pr) correlated with trafficking of Fas/FasL to the cell membrane, indicating that activation of the Fas/FasL apoptotic pathway by par-4 may play an important role in par-4-induced apoptosis (Chakraborty et al. 2001). In contrast, par-4 did not induce apoptosis in the AR-positive epithelial cells (LNCaP and normal prostate epithelial cells). On the basis of our observations that par-4 is a coactivator of $\mathrm{AR}$ and that $\mathrm{AR}$ inhibits par-4-induced apoptosis in PC3 cells (data not shown), we hypothesize that par-4 plus AR activates transcription of genes whose function is to suppress Fas/FasLmediated apoptosis. One candidate, c-FLIP, was found to prevent Fas/FasL-mediated apoptosis by inhibiting caspase-8 activation at the death-inducing signaling complex (Chang et al. 2002), and we recently reported that AR directly targets the c-FLIP gene (Gao et al. 2005). Western blot analysis revealed that the c-FLIP protein levels in cell lines expressing the N-terminal peptide of par-4 were lower than those in the control cell line (Fig. 7A, top panel, lanes 2 and 3 versus lane 1). In contrast, the levels of $\beta$-actin were not affected by expression of the N-terminal peptide of par-4 (Fig. 7A, bottom panel). Northern blot analysis demonstrated that the androgen up-regulated c-FLIP mRNA about twofold
(Fig. 7B, top two panels, lane 2 versus lane 1). Expression of the N-terminal peptide of par-4 inhibited the androgen-induced expression of the c-FLIP gene to $1 \cdot 2$-fold (lane 4 versus lane 3 ) and $1 \cdot 1$-fold (lane 6 versus lane 5) for the cell line \#1 and \#6 respectively. These observations indicate that as a cofactor of AR, par-4 targets the c-FLIP gene. Transient transfection of the c-FLIP reporter plasmid into PC3 cells in the presence of AR approximately doubled luciferase activity over that measured in the absence of $\mathrm{AR}$, and co-expressed par-4 further enhanced this activity (Fig. 7C). Three functional AREs were identified within the c-FLIP gene (Gao et al. 2005). Par-4 did not influence ARE1,2mutated (C61T, G67A, C95T, G101A) c-FLIP reporter gene activity, indicating that the enhancing effect of par-4 on c-FLIP gene expression was AR-dependent (Fig. 7G). We previously demonstrated that AR bound to the c-FLIP promoter regions in the presence of androgen by chromatin immunoprecipitation assay (Gao et al. 2005). The chromatin immunoprecipitation assay also showed the androgen-dependent recruitment of par-4 onto the c-FLIP promoter (Fig. 7D, lane 4 versus lane 3). The products amplified by PCR at the same time from the $\beta$-actin promoter, which served as negative controls, were not changed in response to the addition of androgen (Fig. 7D, lanes 1 and 2). These results indicate that par-4 with AR directly targets the c-FLIP promoter to enhance its gene expression in response to androgens. Thus, apoptosis induced by expression of the N-terminal peptide of par-4 in LNCaP cells might result from the down-regulation of c-FLIP gene expression. Consistent with this conclusion, we observed that only the c-FLIP negative cells underwent apoptosis in the par-4(1-36)-LNCaP cell line (Fig. 6D, panel d).

\section{Loss of c-FLIP expression was essential for the castration-induced apoptosis in the prostate gland}

The cell death that occurs in the prostate gland after castration is one of the typical apoptosis models for studying the morphological and biochemical events

Figure 8 Loss of c-FLIP expression is essential for the castration-induced apoptosis. (A) The prostate gland samples were obtained from the sham-operated mouse (left panel) or from mice 2, 7, 15, or 30 days after castration. The top row shows photomicrographs of the prostate glands of the sham-operated mouse (a) and mice at different times after castration (b-e). Immunohistochemical staining of the prostate glands with anti-AR (second row), anti-par-4 (third row), or anti-c-FLIP (fourth row) antibodies is shown. The negatively stained cells are indicated by white arrows. Panels u-y show the apoptotic cells revealed by TUNEL assay.

TUNEL-positive cells are indicated by white arrows. The insert image shows the double staining of epithelial cells with anti-c-FLIP (red, indicated by a blue arrow) and TUNEL (green, indicated by a white arrow). The bottom row shows immunofluorescent staining of mouse prostate epithelial cells with anti-c-FLIP antibody in the absence (left panel) or presence (right panel) of the antigen $\left(0.4 \mathrm{mg} / \mathrm{ml} \mathrm{c}-\right.$ FLIP $\left._{\mathrm{s}}\right)$. The same slide was stained for the nucleus with DAPI (second panel). The c-FLIP staining and DAPI staining were merged (third panel). Original magnification is $16 x$ for all photomicrographs except the insert $(32 x)$ and the bottom row of images (64x). (B) A model of AR/par-4-mediated survival and apoptosis in prostate cells. In the presence of androgens, AR and par-4 promote c-FLIP gene expression and high levels of c-FLIP inhibit the recruitment of caspase-8 to the Fas/FasL-associated death domain protein (FADD). In the absence of androgens, loss of c-FLIP expression and high levels of par-4 activate Fas/FasL-mediated apoptosis. 


\section{A}

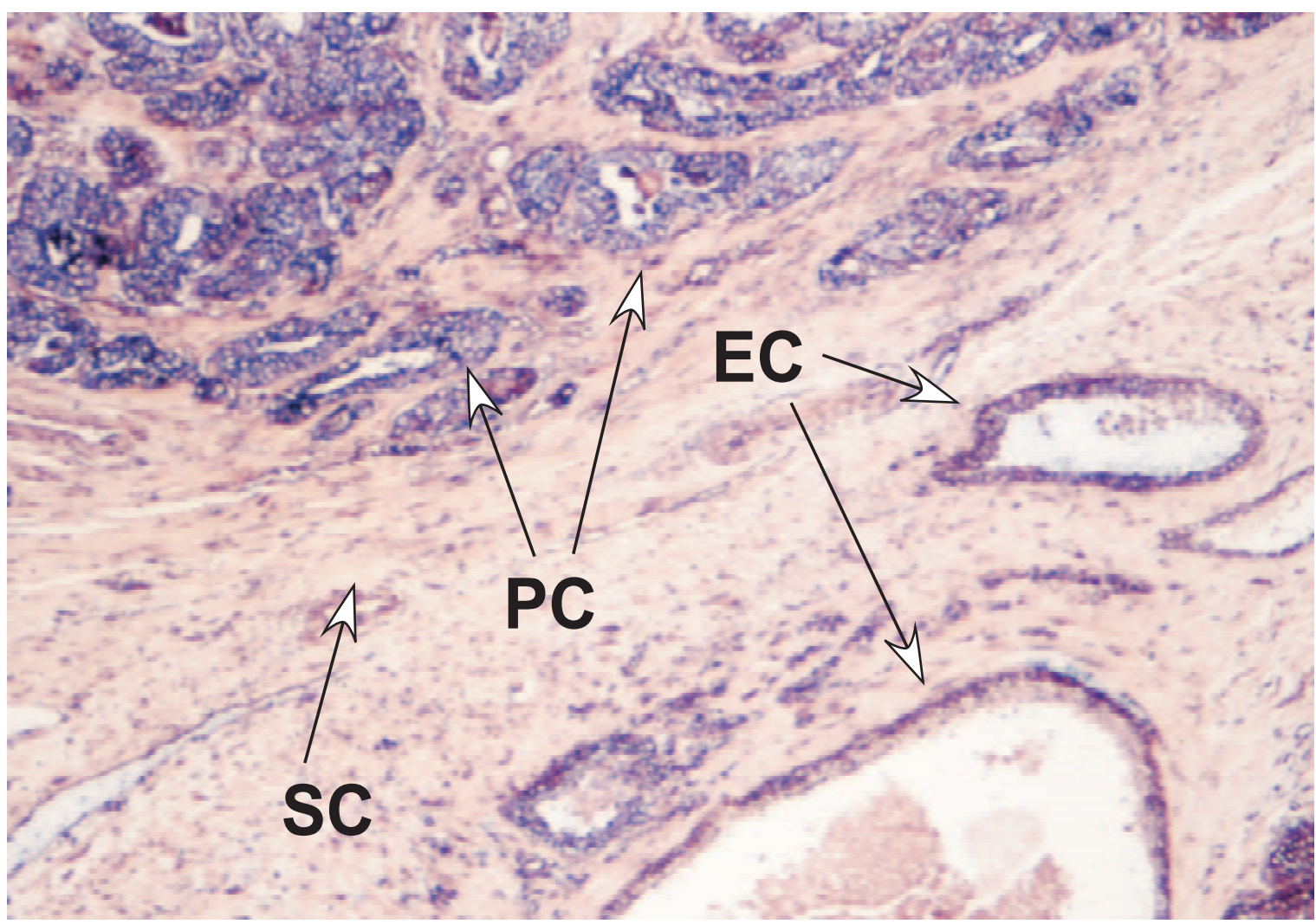

B

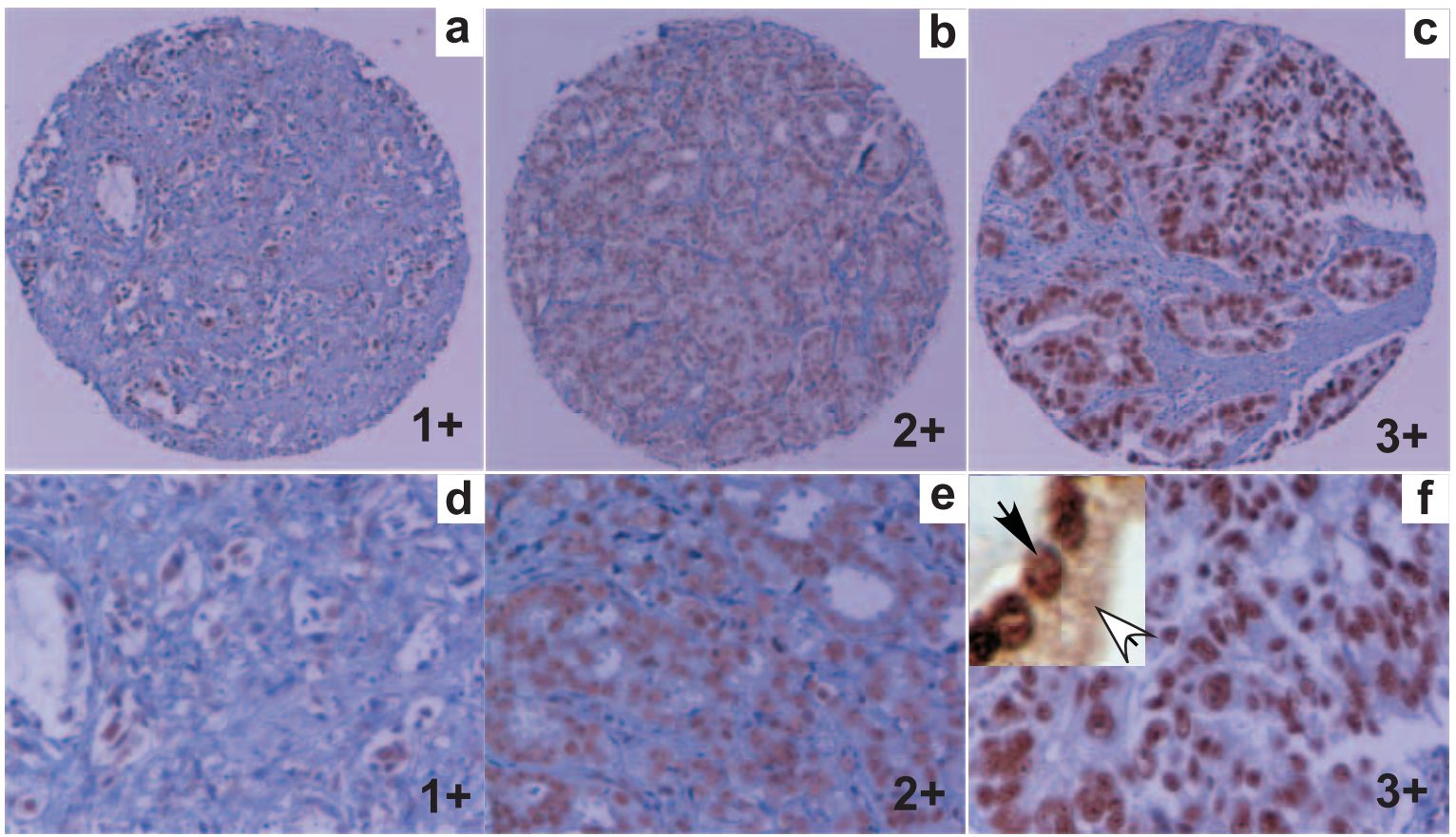


involved in apoptosis (Evans-Storms \& Cidlowski 1995). Although the natural features of castration-induced apoptotic prostate cells have been well documented $(\mathrm{Hu}$ et al. 1998), the mechanism of castration-induced prostate cell death has not been well defined. In order to investigate the role of AR, par-4, and c-FLIP in the castration-induced apoptosis in mouse prostate, castrated male mice were killed at each of 2, 7, 15, and 30 days after operation. The prostate glands gradually changed from well-branched to small and un-branched and were tightly surrounded by several layers of stromal cells (Fig. 8A, top panels a-e). The AR protein levels began to drop 2 days after castration and were very low 7 days after surgery (Fig. 8A, f-j). The par-4 protein stained strongly in the nucleus and weakly in the cytoplasm in epithelial cells, and its levels were induced 2 days after castration (Fig. 8A, panel l) and remained at high levels (panels $\mathrm{m}-\mathrm{o}$ ). Strong c-FLIP signals were detected in the nucleus of the epithelial cells (Fig. 8A, panels p-t). A few $(4 \cdot 3 \% \pm 1 \cdot 3)$ c-FLIP-negative epithelial cells appeared 2 days after castration (Fig. 8A, panel q, indicated by white arrows). Seven days after castration, the number of c-FLIP-negative cells increased substantially $(11 \% \pm 2 \cdot 3)$ (Fig. 8A, panel $r$, indicated by white arrows). The number of c-FLIP negative cells then gradually decreased, reaching basal levels 30 days after castration, and strong c-FLIP signals were then detected in nearly all epithelial cells (Fig. 8A, panel t). The castration-induced apoptotic cells were detected by the TUNEL assay. In control mice, a few apoptotic cells $(0 \cdot 15 \% \pm 0 \cdot 08)$ were found (Fig. 8A, panel u). The number of apoptotic cells $(0 \cdot 57 \% \pm 0 \cdot 09)$ had increased above the control cells 2 days after castration (panel $\mathrm{v}$, indicated by a white arrow), and a large number $(1 \cdot 56 \% \pm 0 \cdot 26)$ was present on the seventh day (panel $w$, indicated by white arrows). Thirty days after castration, the level of apoptotic cells $(0 \cdot 13 \% \pm$ 0.06) was the same as the level of control cells (Fig. 8A, panel y). We performed anti-c-FLIP immunostaining (red) and the TUNEL assay (blue) with the same slides and found that only the c-FLIP-negative cells (indicated by a white arrow) had undergone apoptosis (Fig. 8A, panel $\mathrm{w}$, insert). These results suggest that loss of c-FLIP expression is essential for castration-induced apoptosis. In total, our results indicate that the AR-par-4 pathway controls survival and apoptosis in the prostate gland through regulation of c-FLIP gene expression. The nuclear and cytoplasmic localization of c-FLIP in the prostate epithelial cells was further demonstrated by immunofluorescent staining with anti-c-FLIP antibody and by nuclear staining with DAPI (Fig. 8A, bottom panels). The presence of the antigen (c-FLIP s $_{\mathrm{s}}$ dramatically decreased the immunostaining signals (Fig. 8A, bottom right panel), indicating the specificity of immunohistochemical analysis.

\section{Par-4 was overexpressed in prostate cancer}

Recent studies indicated that par-4 had tumorsuppression activity in mouse prostate gland and its expression was down-regulated in some cancer tissues (Cook et al. 1999, Kogel et al. 2001, Boehrer et al. 2002, Garcia-Cao et al. 2005). To see whether par-4 plays any role in human prostate tumorigenesis, we evaluated the expression of par-4, in correlation with c-Flip expression, in 44 prostate cancer tissues derived from archival prostectomy specimens by in situ hybridization analysis and found that 21 patients with prostate cancer (48\%) had elevated par-4 expression in cancer tissues compared with the adjacent benign prostate tissues (Fig. 9A). Five cases (11\%) showed lower expression of par-4 in tumors compared with adjacent benign tissues. Eighteen cases $(41 \%)$ showed no obvious change in par-4 expression. It has been shown that par-4 expression is induced by various stresses (El-Guendy \& Rangnekar 2003). The observed par-4 over-expression may have resulted from stresses such as hypoxic environment in the prostate cancer tissues. In the same specimens, 22 of $44(50 \%)$ of cases showed increased expression levels for c-FLIP in cancer tissues. Among the $48 \%$ of cases in which par- 4 was over-expressed, $67 \%$ of cases also showed c-FLIP over-expression. On the basis of our observations, these results suggest that upregulation of c-FLIP expression might result from up-regulation of par-4 expression. Because the NF-кB signaling pathway also up-regulates c-FLIP gene expression (Micheau et al. 2001), other signals might be responsible for c-FLIP over-expression in the other prostate cancer cases.

The c-FLIP expression was also examined in prostate tissue microarray by immunohistochemistry. In the tissue microarray, 16 cases are hormone resistant, 23 cases are hormone naive patients, either from prostatectomy cases or transurethral resection specimens. Nine

Figure 9 (A) Over-expression of par-4 in prostate cancer. The slides were hybridized with digoxigenin-labeled RNA probes of par-4. A positive signal is purple or blue, and the negative signal is colorless or light pink. EC, benign prostate epithelial cells; SC, prostate stromal cells; PC, prostate cancer cells. (B) Enhanced c-FLIP expression in androgen-resistant prostate cancer. The c-FLIP expression was examined in prostate tissue microarray by immunohistochemistry with anti-c-FLIP antibody. The intensity of c-FLIP expression was scored semi-quantitatively as $1+$ for weak expression (panels $a, d), 2+$ for moderate expression (panels b, e), and $3+$ for strong expression (panels c, f). The panels $d, e, f(20 \times)$ show the high power amplification of panels $a, b, c(4 \times)$. The insert in panel f shows $64 \times$ amplification and the nuclear and cytoplasmic localization of c-FLIP is indicated by black and white arrows respectively. 
cases are from neoadjuvant therapy patients. c-FLIP was expressed in both benign and cancerous prostatic epithelium and localized in both the nucleus (Fig. 9B, panel $f$, insert, indicated by a black arrow) and the cytoplasm (indicated by a white arrow). The intensity of c-Flip expression was scored semi-quantitatively as $1+$ for weak expression, $2+$ for moderate expression, and $3+$ for strong expression (Fig. 9B). Of the 16 hormone resistant cases, $3(19 \%)$ showed weak $1+$ expression and $13(81 \%)$ showed $2+$ or $3+$ staining. Of 23 hormone naive cases, $11(48 \%)$ showed weak $1+$ expression and $13(52 \%)$ showed $2+$ or $3+$ staining. Similarly, of the 9 cases with neoadjuvant therapy, $4(44 \%)$ showed weak $1+$ expression and $4(56 \%)$ showed $2+$ or $3+$ staining. These results indicate that there is a stronger c-Flip expression in hormone-resistant cases than in others including hormone naive or neoadjuvant therapy treated patients.

\section{Discussion}

In this study, we found evidence that proapoptotic factor par-4 is an AR-interacting protein that modulates the transcriptional activities of $\mathrm{AR}$ by enhancing AR-ARE interaction. Further analysis indicated that $\mathrm{AR}$ and par-4 directly targeted the c-FLIP gene. A decrease of c-FLIP expression by the dominant negative par-4 in LNCaP cells or loss of c-FLIP expression induced by castration in mice led to apoptosis. Collectively, our results indicate that modulation of c-FLIP expression by $\mathrm{AR}$ and par-4 plays an important role in prostate cell survival and apoptosis.

\section{The structure and functions of par-4}

Par-4 has a leucine zipper domain that spans the region between amino acid residues 301 and 342 (Dutta et al. 2001). The leucine zipper structure is packed together in a parallel $\alpha$-helix with the conserved leucine residues located on one side of each helix (Johnson \& McKnight 1989). The leucine zipper domain has been found in many transcription factors, including myc, fos, and jun, and is essential for the formation of homodimeric and heterodimeric complexes. Consistent with previous observations (Sells et al. 1994, Nessler-Menardi et al. 2000, Moscat \& Diaz-Meco 2003, Culig et al. 2004), we found that deletion of the $\mathrm{C}$-terminal part (amino acid residues 269-342) dramatically decreased par-4's interaction with itself, and that the $\mathrm{C}$-terminal parts (amino acid residues 137-342 and 201-342) strongly interacted with themselves. The regions outside the C-terminal part (amino acid residues 269-342) also contributed to par-4's interaction with itself, but to a much lesser extent. In contrast to its function in apoptosis, the C-terminal leucine zipper region (amino acid 202-342) of par-4 was not required for its cofactor, AR-dependent, transcription function. These results suggest that par-4's cofactor activity can be separated from its ability to stimulate apoptosis.

Intermolecular interaction plays important roles in regulating the functions of various transcription factors (Hupp et al. 1995, Jayaraman \& Prives 1995, Liu et al. 1998, Liu et al. 2003). We found a novel interaction between the $\mathrm{N}$-terminal part (amino acid residues 1-36) and the C-terminal part (amino acid residues 269-342) of par-4. This interaction strongly inhibited par- 4 's interaction with itself and with AR. The intermolecular interaction was not apparently required for the par-4-mediated enhancement of AR-driven gene expression because deletion of amino acid residues 1-36 or 269-342 had no effect. It is not known whether the intermolecular interaction is involved in par-4-mediated apoptosis. The N-terminal peptide of par-4 did not interact with $\mathrm{AR}$, and deletion of amino acid residues 1-36 from par-4 did not affect the par-4-mediated enhancement of AR-driven transcription, but strongly interacted with the $\mathrm{C}$-terminal 270 amino acid residues. On the basis of these observations, we believe that the dominant-negative effect of the N-terminal peptide of par-4 is mediated through its interaction with the C-terminal part of par-4.

\section{Par-4 functions as a new cofactor for AR}

More than 10 factors have been shown to interact with the $\mathrm{DBD}$ of $\mathrm{AR}$ and to modulate $\mathrm{AR}$-driven transcription (ww2.mcgill.ca/androgendb/ARinteract.pdf). The molecular mechanisms of the action of these factors on AR-driven gene expression are largely unknown. Some factors, such as prostate epithelium-specific ETS transcription factor (PDEF) (Oettgen et al. 2000) and octamer binding transcription factor (OCT)-1/2 (Prefontaine et al. 1999, Gonzalez \& Robins 2001), have sequence-specific DNA-binding activity and might synergistically interact with DNA in the presence of AR. It was also reported that c-jun interacted with the DBD of $\mathrm{AR}$ and inhibited AR-ARE interaction (Sato et al. 1997). Our finding that the DBD of AR (containing two zinc fingers) mediates its interaction with par-4 is reminiscent of the WT1-par-4 interaction previously reported (Johnstone et al. 1996). In that study, the four zinc fingers of WT1 were shown to interact with par-4. This par-4-WT1 interaction plays a role in par-4mediated repression of transcription of WT1. It thus appears that par-4 may interact with a number of cellular proteins via their zinc finger-containing domains. Consistent with this conclusion, we observed that par-4 enhanced estrogen receptor $\alpha$-, glucocorticoid receptor-, and progesterone receptor $\beta$-driven gene expression (data not shown). Par-4 was shown to repress reporter gene expression when tethered to DNA via the GAL4 DNA-binding domain, suggesting that it has the 
repression domain (Johnstone et al. 1996, Babichev et al. 2001). It was also demonstrated that transactivation of the alternative splicing isoform of WT1 was dependent on a specific interaction with par-4, and a mutation in WT1 found in Wilms' tumor disturbs this interaction and the function of the activation of WT1 (Richard et al. 2001). Induced par-4 expression was strongly correlated with the ability of WT1 to regulate cell survival and proliferation. Thus, the functions of par-4 as coactivator or co-repressor might depend on its interacting protein partners, cell type, the promoter context, or some combination of these. The mechanisms that control the activation and repression functions of par-4 have yet to be fully elucidated. Our observations suggest that the par-4-mediated enhancement of AR (possibly the WT1 isoform) transaction might occur through an increase in $\mathrm{AR}-\mathrm{ARE}$ interaction.

\section{AR and par-4 target c-FLIP gene expression to control apoptosis in the prostate gland}

We previously demonstrated that the AR pathway directly targets the c-FLIP gene, and we identified three functional AREs in the c-FLIP promoter (Gao et al. 2005). Here, we showed that par-4 functions as an AR cofactor for androgen-driven c-FLIP gene expression. This conclusion was supported by the following three experiments. First, we observed that par-4 enhanced androgendriven c-FLIP promoter activity in the transient trasnfection assay. Secondly, chromatin immunoprecipitation analysis indicated that par-4 directly bound to the promoter region of the c-FLIP gene in response to the androgen treatment, suggesting that it directly targeted the c-FLIP gene. Thirdly, expression of the dominantnegative par-4 inhibited c-FLIP gene expression.

The androgen pathway exerts a protective effect in the prostate gland (Hakimi et al. 1996) and in androgen-sensitive human prostate cancer LNCaP cells (Coffey et al. 2002). Although the mechanisms underlying these effects have not been clearly defined, androgen's effects on both proapoptotic and antiapoptotic gene expression have been demonstrated (Coffey et al. 2002). We observed that AR levels gradually decreased in all epithelial cells following castration and reached the lowest level 7 days after castration. In contrast, par-4 levels increased simultaneously in all epithelial cells after castration and then stayed at high levels. It was surprising that a few epithelial cells lost c-FLIP expression following castration. Most interestingly and significantly, apoptosis was only detected in the epithelial cells that did not express c-FLIP. Transcription is traditionally considered as a graded process with promoter activity changing proportionally in response to various signals. The expression of the AR gene in response to castration is compatible with this model. But, recent work on the nature of enhancer action suggests a binary model of transcription control (Fiering et al. 2000). The binary model implies that enhancers act in a stochastic fashion to increase the probability that a regulated gene will be transcribed. This model would predict a non-uniform reduction in c-FLIP gene expression, with castration resulting in a decreased fraction of cells that express c-FLIP gene (at or near wild-type levels) relative to the fraction of cells that do not express c-FLIP gene at all. Thus, castrationinduced apoptosis could go on continuously in individual cells at various times by controlling c-FLIP expression. Thirty days after castration, the c-FLIP levels were very high in all epithelial cells and then apoptosis largely ceased, although par-4 levels were very high. These results suggest that expression of the c-FLIP gene is not solely controlled by the androgen pathway but also by other signals such as the NF-кB pathway (Micheau et al. 2001). The combination of the AR pathway and these signals might determine the levels of c-FLIP in prostate epithelial cells.

It is well documented that c-FLIP functions as a potent inhibitor of the Fas/FasL-induced apoptosis and that par-4 induces apoptosis. Our present data provide a framework to understand how androgen can determine survival and apoptosis in the prostate gland (Fig. 8B). Specifically, in the presence of androgens, both AR and par-4 act as transcriptional activators or co-activators for the expression of genes whose protein inhibits apoptosis (such as c-FLIP) or promotes cell survival. However, in the absence of androgens (such as after castration), c-FLIP expression is lost, and par-4 induces apoptosis by activation of Fas/FasL-mediated apoptosis. This model also explains why ectopic expression of par-4 induced apoptosis of AR-negative prostate cancer cells but not of AR-positive prostate cancer and normal prostate epithelial cells (Chakraborty et al. 2001). On the basis of our observations, we speculate that the androgenindependent expression of the c-FLIP gene would contribute to the androgen-independent growth of prostate cancer cells. Consistent with this possibility, we observed enhanced c-FLIP expression in the androgenresistant prostate cancer and over-expression of the c-FLIP gene enhanced the androgen-independent growth of LNCaP xenographs in nude mice (Gao et al. 2005). Given that par-4 plays a role in enhancing apoptosis in response to various stimuli and functions as a novel transcriptional cofactor of AR, the study of par-4 regulation mechanisms will help us to understand par-4's roles in cancer and neuronal diseases.

\section{Acknowledgements}

We thank Ms Ann Sutton for the critical editorial review and Drs Douglas Boyd and David J McConkey for scientific comments on the manuscript. This work was 
supported in part by a grant (1R01 DK065156 01) to Z W from the National Institute of Diabetes and Digestive and Kidney Diseases and the National Institutes of Health and by a Cancer Center Support Core grant (CA16672) to M D Anderson Cancer Center from the National Cancer Institute, National Institutes of Health. The authors declare that there is no conflict of interest that would prejudice the impartiality of this scientific work.

\section{References}

Babichev VN, Marova EI, Kuznetsova TA, Adamskaya EI, Shishkina IV \& Yu Kasumova S 2001 Role of sex hormones in development of pituitary adenoma. Bulletin of Experimental Biology and Medicine 131 309-311.

Bentel JM \& Tilley WD 1996 Androgen receptors in prostate cancer. Fournal of Endocrinology 151 1-11.

Boehrer S, Chow KU, Beske F, Kukoc-Zivojnov N, Puccetti E, Ruthardt M, Baum C, Rangnekar VM, Hoelzer D, Mitrou PS et al. 2002 In lymphatic cells par-4 sensitizes to apoptosis by down-regulating bcl-2 and promoting disruption of mitochondrial membrane potential and caspase activation. Cancer Research 62 1768-1775.

Brinkmann AO, Blok LJ, de Ruiter PE, Doesburg P, Steketee K, Berrevoets CA \& Trapman J 1999 Mechanisms of androgen receptor activation and function. Fournal of Steroid Biochemistry and Molecular Biology 69 307-313.

Chakraborty M, Qiu SG, Vasudevan KM \& Rangnekar VM 2001 Par-4 drives trafficking and activation of Fas and Fasl to induce prostate cancer cell apoptosis and tumor regression. Cancer Research $617255-7263$.

Chang DW, Xing Z, Pan Y, Algeciras-Schimnich A, Barnhart BC, Yaish-Ohad S, Peter ME \& Yang X 2002 c-FLIP(L) is a dual function regulator for caspase-8 activation and CD95-mediated apoptosis. EMBO fournal 21 3704-3714.

Cheema SK, Mishra SK, Rangnekar VM, Tari AM, Kumar R \& Lopez-Berestein G 2003 Par-4 transcriptionally regulates Bcl-2 through a WT1-binding site on the bcl-2 promoter. Fournal of Biological Chemistry 278 19995-20005.

Claessens F, Alen P, Devos A, Peeters B, Verhoeven G \& Rombauts W 1996 The androgen-specific probasin response element 2 interacts differentially with androgen and glucocorticoid receptors. Fournal of Biological Chemistry 271 19013-19016.

Cleutjens KB, van Eekelen CG, van der Korput HA, Brinkmann AO \& Trapman J 1996 Two androgen response regions cooperate in steroid hormone regulated activity of the prostate-specific antigen promoter. Fournal of Biological Chemistry $2716379-6388$.

Coffey RN, Watson RW, O’Neill AJ, McEleny K \& Fitzpatrick JM 2002 Androgen-mediated resistance to apoptosis. Prostate 53 300-309.

Cook J, Krishnan S, Ananth S, Sells SF, Shi Y, Walther MM, Linehan WM, Sukhatme VP, Weinstein MH \& Rangnekar VM 1999 Decreased expression of the pro-apoptotic protein Par-4 in renal cell carcinoma. Oncogene 18 1205-1208.

Craft N, Chor C, Tran C, Belldegrun A, DeKernion J, Witte ON, Said J, Reiter RE \& Sawyers CL 1999 Evidence for clonal outgrowth of androgen-independent prostate cancer cells from androgen-dependent tumors through a two-step process. Cancer Research 59 5030-5036.

Culig Z, Comuzzi B, Steiner H, Bartsch G \& Hobisch A 2004 Expression and function of androgen receptor coactivators in prostate cancer. Fournal of Steroid Biochemistry and Molecular Biology 92 265-271.

Denmeade SR, Lin XS \& Isaacs JT 1996 Role of programmed (apoptotic) cell death during the progression and therapy for prostate cancer. Prostate 28 251-265.

Diaz-Meco MT, Municio MM, Frutos S, Sanchez P, Lozano J, Sanz L \& Moscat J 1996 The product of par-4, a gene induced during apoptosis, interacts selectively with the atypical isoforms of protein kinase C. Cell 86 777-786.

Dutta K, Alexandrov A, Huang H \& Pascal SM 2001 pH-induced folding of an apoptotic coiled coil. Protein Science 10 2531-2540.

El-Guendy N \& Rangnekar VM 2003 Apoptosis by Par-4 in cancer and neurodegenerative diseases. Experimental Cell Research 283 51-66.

El-Guendy N, Zhao Y, Gurumurthy S, Burikhanov R \& Rangnekar VM 2003 Identification of a unique core domain of par-4 sufficient for selective apoptosis induction in cancer cells. Molecular and Cellular Biology 23 5516-5525.

Evans-Storms RB \& Cidlowski JA 1995 Regulation of apoptosis by steroid hormones. Fournal of Steroid Biochemistry and Molecular Biology 53 1-8.

Fiering S, Whitelaw E \& Martin DI 2000 To be or not to be active: the stochastic nature of enhancer action. Bioessays 22 381-387.

Fondell JD, Ge H \& Roeder RG 1996 Ligand induction of a transcriptionally active thyroid hormone receptor coactivator complex. PNAS 93 8329-8333.

Gao S, Lee P, Wang H, Gerald W, Adler M, Zhang L, Wang YF \& Wang Z 2005 The androgen receptor directly targets the cellular Fas/FasL-associated death domain protein-like inhibitory protein gene to promote the androgen-independent growth of prostate cancer cells. Fournal of Molecular Endocrinology 19 1792-1802.

Garcia-Cao I, Lafuente MJ, Criado LM, Diaz-Meco MT, Serrano M \& Moscat J 2003 Genetic inactivation of Par4 results in hyperactivation of NF-kappaB and impairment of JNK and p38. EMBO Reports 4 307-312.

Garcia-Cao I, Duran A, Collado M, Carrascosa MJ, Martin-Caballero J, Flores JM, Diaz-Meco MT, Moscat J \& Serrano M 2005 Tumour-suppression activity of the proapoptotic regulator Par4. EMBO Reports 6 577-583.

Gelmann EP 2002 Molecular biology of the androgen receptor. Journal of Clinical Oncology $203001-3015$.

Gonzalez MI \& Robins DM 2001 Oct-1 preferentially interacts with androgen receptor in a DNA-dependent manner that facilitates recruitment of SRC-1. Fournal of Biological Chemistry 276 6420-6428.

Guo Q Fu W, Xie J, Luo H, Sells SF, Geddes JW, Bondada V, Rangnekar VM \& Mattson MP 1998 Par-4 is a mediator of neuronal degeneration associated with the pathogenesis of Alzheimer disease. Nature Medicine 4 957-962.

Gurumurthy S \& Rangnekar VM 2004 Par-4 inducible apoptosis in prostate cancer cells. Fournal of Cellular Biochemistry 91 504-512.

Hakimi JM, Rondinelli RH, Schoenberg MP \& Barrack ER 1996 Androgen-receptor gene structure and function in prostate cancer. World Joumal of Urology 14 329-337.

Heinlein CA \& Chang C 2002 Androgen receptor (AR) coregulators: an overview. Endocrine Reviews 23 175-200.

Hosohata K, Li P, Hosohata Y, Qin J, Roeder RG \& Wang Z 2003 Purification and identification of a novel complex which is involved in androgen receptor-dependent transcription. Molecular and Cellular Biology 23 7019-7029.

Hu Z, Ito T, Yuri K, Xie C, Ozawa H \& Kawata M 1998 In vivo time course of morphological changes and DNA degradation during the degeneration of castration-induced apoptotic prostate cells. Cell and Tissue Research 294 153-160.

Hupp TR, Sparks A \& Lane DP 1995 Small peptides activate the latent sequence-specific DNA binding function of p53. Cell 83 237-245. 
Janne OA, Moilanen AM, Poukka H, Rouleau N, Karvonen U, Kotaja N, Hakli M \& Palvimo JJ 2000

Androgen-receptor-interacting nuclear proteins. Biochemical Society Transactions 28 401-405.

Jayaraman J \& Prives C 1995 Activation of p53 sequence-specific DNA binding by short single strands of DNA requires the p53 C-terminus. Cell 81 1021-1029.

Johnson PF \& McKnight SL 1989 Eukaryotic transcriptional regulatory proteins. Annual Review of Biochemistry 58 799-839.

Johnstone RW, See RH, Sells SF, Wang J, Muthukkumar S, Englert C, Haber DA, Licht JD, Sugrue SP, Roberts T et al. 1996 A novel repressor, par-4, modulates transcription and growth suppression functions of the Wilms' tumor suppressor WT1. Molecular and Cellular Biology 16 6945-6956.

Kogel D, Plottner O, Landsberg G, Christian S \& Scheidtmann KH 1998 Cloning and characterization of Dlk, a novel serine/threonine kinase that is tightly associated with chromatin and phosphorylates core histones. Oncogene 17 2645-2654.

Kogel D, Reimertz C, Mech P, Poppe M, Fruhwald MC, Engemann H, Scheidtmann KH \& Prehn JH 2001 Dlk/ZIP kinase-induced apoptosis in human medulloblastoma cells: requirement of the mitochondrial apoptosis pathway. British Fournal of Cancer 85 1801-1808.

Li P, Yu X, Ge K, Melamed J, Roeder RG \& Wang Z 2002 Heterogeneous expression and functions of androgen receptor co-factors in primary prostate cancer. American Foumal of Pathology 161 1467-1474.

Liu D, Ishima R, Tong KI, Bagby S, Kokubo T, Muhandiram DR, Kay LE, Nakatani Y \& Ikura M 1998 Solution structure of a TBP-TAF(II)230 complex: protein mimicry of the minor groove surface of the TATA box unwound by TBP. Cell 94 573-583.

Liu GZ, Wang H \& Wang Z 2003 Identification of a highly conserved domain in the androgen receptor that suppresses the DNA-binding domain-DNA interactions. Fournal of Biological Chemistry 278 14956-14960.

Mattson MP, Duan W, Chan SL \& Camandola S 1999 Par-4: an emerging pivotal player in neuronal apoptosis and neurodegenerative disorders. Fournal of Molecular Neuroscience 13 17-30.

Meyer F, Moore L, Bairati I, Lacombe L, Tetu B \& Fradet Y 1999 Neoadjuvant hormonal therapy before radical prostatectomy and risk of prostate specific antigen failure. Fournal of Urology $1622024-2028$

Micheau O, Lens S, Gaide O, Alevizopoulos K \& Tschopp J 2001 NF-kappaB signals induce the expression of c-FLIP. Molecular and Cellular Biology 21 5299-5305.

Miyoshi Y, Ishiguro H, Uemura H, Fujinami K, Miyamoto H, Kitamura H \& Kubota Y 2003 Expression of AR associated protein 55 (ARA55) and androgen receptor in prostate cancer. Prostate 56 280-286.

Moscat J \& Diaz-Meco MT 2003 Par-4 keeps the atypical PKCs at bay. Cell Cycle 2 71-72.

Nessler-Menardi C, Jotova I, Culig Z, Eder IE, Putz T, Bartsch G \& Klocker H 2000 Expression of androgen receptor coregulatory proteins in prostate cancer and stromal-cell culture models. Prostate 45 124-131.
Oettgen P, Finger E, Sun Z, Akbarali Y, Thamrongsak U, Boltax J, Grall F, Dube A, Weiss A, Brown L et al. 2000 PDEF, a novel prostate epithelium-specific ets transcription factor, interacts with the androgen receptor and activates prostate-specific antigen gene expression. Journal of Biological Chemistry 275 1216-1225.

Pear WS, Miller JP, Xu L, Pui JC, Soffer B, Quackenbush RC, Pendergast AM, Bronson R, Aster JC, Scott ML et al. 1998 Efficient and rapid induction of a chronic myelogenous leukemia-like myeloproliferative disease in mice receiving P210 bcr/abl-transduced bone marrow. Blood 92 3780-3792.

Powell IJ, Tangen CM, Miller GJ, Lowe BA, Haas G, Carroll PR, Osswald MB, De Vere White R, Thompson IM Jr \& Crawford ED 2002 Neoadjuvant therapy before radical prostatectomy for clinical T3/T4 carcinoma of the prostate: 5-year follow-up, Phase II Southwest Oncology Group Study 9109. Fournal of Urology $1682016-2019$.

Prefontaine GG, Walther R, Giffin W, Lemieux ME, Pope L \& Hache RJ 1999 Selective binding of steroid hormone receptors to octamer transcription factors determines transcriptional synergism at the mouse mammary tumor virus promoter. Fournal of Biological Chemistry 274 26713-26719.

Richard DJ, Schumacher V, Royer-Pokora B \& Roberts SG 2001 Par-4 is a coactivator for a splice isoform-specific transcriptional activation domain in WT1. Genes and Development 15 328-339.

Sato N, Sadar MD, Bruchovsky N, Saatcioglu F, Rennie PS, Sato S, Lange PH \& Gleave ME 1997 Androgenic induction of prostate-specific antigen gene is repressed by protein-protein interaction between the androgen receptor and AP-1/c-Jun in the human prostate cancer cell line LNCaP. Fournal of Biological Chemistry 272 17485-17494.

Schuur ER, Henderson GA, Kmetec LA, Miller JD, Lamparski HG \& Henderson DR 1996 Prostate-specific antigen expression is regulated by an upstream enhancer. Fournal of Biological Chemistry 271 7043-7051.

Sells SF, Wood DP Jr, Joshi-Barve SS, Muthukumar S, Jacob RJ, Crist SA, Humphreys S \& Rangnekar VM 1994 Commonality of the gene programs induced by effectors of apoptosis in androgen-dependent and -independent prostate cells. Cell Growth and Differentiation 5 457-466.

Sells SF, Han SS, Muthukkumar S, Maddiwar N, Johnstone R, Boghaert E, Gillis D, Liu G, Nair P, Monnig S et al. 1997 Expression and function of the leucine zipper protein Par-4 in apoptosis. Molecular and Cellular Biology 17 3823-3832.

Wang Z \& Roeder RG 1997 Three human RNA polymerase III-specific subunits form a subcomplex with a selective function in specific transcription initiation. Genes and Development 11 1315-1326.

Yu H, Diamandis EP, Zarghami N \& Grass L 1994 Induction of prostate specific antigen production by steroids and tamoxifen in breast cancer cell lines. Breast Cancer Research and Treatment 32 291-300.

Received in final form 7 February 2006 Accepted 6 March 2006

Made available online as an Accepted Preprint 27 March 2006 